\title{
Belief Inducibility and Informativeness
}

Citation for published version (APA):

Herings, P. J-J., Karos, D., \& Kerman, T. (2020). Belief Inducibility and Informativeness. Maastricht University, Graduate School of Business and Economics. GSBE Research Memoranda No. 027 https://doi.org/10.26481/umagsb.2020027

Document status and date:

Published: 13/10/2020

DOI:

10.26481/umagsb.2020027

Document Version:

Publisher's PDF, also known as Version of record

\section{Please check the document version of this publication:}

- A submitted manuscript is the version of the article upon submission and before peer-review. There can be important differences between the submitted version and the official published version of record.

People interested in the research are advised to contact the author for the final version of the publication, or visit the DOI to the publisher's website.

- The final author version and the galley proof are versions of the publication after peer review.

- The final published version features the final layout of the paper including the volume, issue and page numbers.

Link to publication

\footnotetext{
General rights rights.

- You may freely distribute the URL identifying the publication in the public portal. please follow below link for the End User Agreement:

www.umlib.nl/taverne-license

Take down policy

If you believe that this document breaches copyright please contact us at:

repository@maastrichtuniversity.nl

providing details and we will investigate your claim.
}

Copyright and moral rights for the publications made accessible in the public portal are retained by the authors and/or other copyright owners and it is a condition of accessing publications that users recognise and abide by the legal requirements associated with these

- Users may download and print one copy of any publication from the public portal for the purpose of private study or research.

- You may not further distribute the material or use it for any profit-making activity or commercial gain

If the publication is distributed under the terms of Article $25 \mathrm{fa}$ of the Dutch Copyright Act, indicated by the "Taverne" license above, 


\section{Maastricht University}

P. Jean-Jacques Herings, Dominik Karos, Toygar Kerman

Belief Inducibility and Informativeness

$\mathrm{RM} / 20 / 027$

ISSN: $2666-8807$

\section{GSBE}

Maastricht University School of Business and Economics

Graduate School of Business and Economics

P.O Box 616

NL- 6200 MD Maastricht

The Netherlands 


\title{
Belief Inducibility and Informativeness
}

\author{
P. Jean-Jacques Herings* Dominik Karos $^{\dagger \ddagger}$ Toygar Kerman $\$$
}

\author{
October 12,2020
}

\begin{abstract}
We consider a group of receivers who share a common prior on a finite state space and who observe private correlated signals that are contingent on the true state of the world. We show that, while necessary, Bayes plausibility is not sufficient for a distribution over posterior belief vectors to be inducible, and we provide a characterization of inducible distributions. We classify communication strategies as minimal, direct, and language independent, and show that any inducible distribution can be induced by a language independent communication strategy (LICS). We investigate the role of the different classes of communication strategies for the amount of higher order information that is revealed to receivers. We show that the least informative communication strategy which induces a fixed distribution over posterior belief vectors lies in the relative interior of the set of all language independent communication strategies which induce that distribution.
\end{abstract}

Keywords: Information Design, Inducible Distributions, Informativeness.

\section{Introduction}

In any economic model which involves a group of agents and has a payoff structure that depends on the posterior beliefs of the agents, one of the essential questions is "Which distributions over posterior beliefs of agents can be induced?" In their seminal paper,

*Department of Economics, Maastricht University, P.O. Box 616, 6200 MD, Maastricht, The Netherlands. p.herings@maastrichtuniversity.nl

${ }^{\dagger}$ The author gratefully acknowledges funding by the ERC, Project Number 747614 .

$\ddagger$ Center for Mathematical Economics, Bielefeld University, Postfach 100131, 33501 Bielefeld, Germany. dominik.karos@uni-bielefeld.de

$\S$ corresponding author

IDepartment of Economics, Maastricht University, P.O. Box 616, 6200 MD, Maastricht, The Netherlands.t.kerman@maastrichtuniversity.nl 
Kamenica and Gentzkow (2011) consider communication between a sender and a receiver who share a common prior and show that the only restriction on the set of inducible distributions over posterior belief vectors is Bayes plausibility: the expected posterior belief is equal to the prior. ${ }^{1}$ It follows from their insight that Bayes plausibility and identical beliefs is necessary and sufficient in the case of multiple receivers and public communication, that is, when messages are perfectly correlated. Yet, in this case the set of inducible distributions over posteriors is very limited since all receivers have the same ex-post belief. In the present paper we are interested in private communication, which, in contrast, enables the sender to achieve a richer belief space; however, Bayes plausibility is no longer sufficient for inducibility. In other words, there are distributions which are Bayes plausible for every agent, but are not induced by any communication strategy. The first question we answer is: what additional conditions are required for the existence of such a communication strategy?

Another aspect which is important for both the sender and receivers is the informativeness of a communication strategy. While the informativeness criterion which is commonly employed in the information design literature is the one of Blackwell (1953), we consider a different definition of informativeness: we compare the informativeness of communication strategies not in terms of ex-post beliefs but in terms of "knowledge" about the true state and the signal realization in the sense of Hintikka (1962). A signal realization induces information partitions that determine what a receiver knows about another receiver's knowledge of the true state and the signal realization. This becomes particularly important if the sender wishes to restrict the information receivers have about each other. The second main question we answer is: what types of communication strategies are the least informative?

We consider a sender who commits to a communication strategy that sends private correlated messages to the receivers. Receivers know the joint the distribution of signals, that is vectors of messages, but they only observe their own private message from the signal realization. We first show that there are posterior belief vectors, which the sender cannot achieve with positive probability. More precisely, for a given posterior belief vector, there exists a communication strategy that induces a distribution which puts positive weight on it if and only if there exists a state which is deemed possible by all receivers according to this belief vector. As an example, consider an operative who follows Machiavelli's advice divide et impera and, thus, wants to create political unrest in a foreign country by implementing a very heterogeneous belief vector. Suppose that there are only two states, say blue and red. Then it is impossible for the operative to implement a distribution that puts positive weight on a posterior belief vector in which one receiver believes the state is blue with probability 1 and another receiver beliefs that the state is red with probability 1. At the same time, a posterior belief vector in which the first receiver's belief that the state is blue is equal to 1 , and the second receiver's belief is arbitrarily close to 0 can be

\footnotetext{
${ }^{1}$ This is also known as the martingale property.
} 
achieved with positive probability.

We next define particular classes of communication strategies. We first consider minimal communication strategies under which distinct signals lead to distinct posterior belief vectors. While this ensures that no two signals implement the same posterior belief vector, there might still be individual receivers for whom different messages lead to the same posterior. If for each receiver every posterior is induced by a unique message, the communication strategy is called direct. If, additionally, the sent messages are themselves posteriors such that each message induces itself, we call the communication strategies language independent (LICS). Here, a sender simply tells the receivers what belief they should have, and the messages are sent with probabilities such receivers will believe the message. We show that restricting attention to language independent communication strategies is without loss of generality, that is, any posterior distribution that can be induced, can be induced by an LICS.

As mentioned before, in the presence of multiple receivers Bayes plausibility is necessary but not sufficient for a distribution to be inducible. We characterize the set of inducible distributions of posteriors by showing that a Bayes plausible distribution is inducible if and only if there exists a matrix $p$ with non-negative entries which satisfies a particular system of linear equations. In particular, the set of matrices that satisfy these equations is a convex polytope, which implies that the set of language independent communication strategies that induce a given distribution over posterior belief vectors is a convex polytope as well.

We next explore the informativeness of different communication strategies which induce the same distribution of posterior beliefs: the message a receiver obtains reveals not only information about the true state of the world, but also about the information that other receivers have. Let's return to our operative who wants to create chaos in a foreign country. If one receiver knew that another receiver knew whether the true state is red or blue, he might decide not to engage in an argument at all. Thus, our operative might want to reveal as little information as possible to any receiver about what other receivers know. As an example suppose that before the operative engages, two receivers believe that either state might be true with probability $1 / 2$. Suppose the operative engages in private communication with both and sends signals as follows.

\begin{tabular}{c|ccc}
$\pi^{\prime}$ & $(m, r)$ & $(m, b)$ & $(x, x)$ \\
\hline Red & $\frac{1}{2}$ & 0 & $\frac{1}{2}$ \\
Blue & 0 & $\frac{1}{2}$ & $\frac{1}{2}$
\end{tabular}

In this case receiver 2 knows that the true state is red if he observes $r$, he knows the true state is blue if he observes $b$, and he learns nothing if he observes $x$. Agent 1 never learns anything about the true state. If he observes $m$, however, he knows that receiver 2 knows the true state. If the sender would replace $m$ by $x$, receiver 1 would not learn anything at all. 
This example illustrates that the knowledge a receiver has about the true state and the signal realization can differ among communication strategies under which all receivers have an identical distribution over posterior belief vectors. In particular, a receiver may have different knowledge about another receiver's knowledge about the true state and the signal realization. It is then natural to ask what types of communication strategies that induce the same distribution restrict this knowledge the most. In the example above, different messages might lead to the same posterior belief but to different higher order knowledge. By employing direct or even language independent communication strategies we could avoid this issue. But even then: not all language independent communication strategies reveal the same amount of information. To make this more precise, we define information correspondences that describe what receivers know about the true state and the true posterior belief vector (instead of the signal realization), where we call a tuple of a state and a posterior belief vector a posterior history. A communication strategy is more informative than another if for every receiver, every state, and every signal that can occur in this state, the set of posterior histories that the receiver deems possible is smaller under the former than under the latter. We prove that for any inducible distribution over posterior belief vectors the least informative communication strategies that induce it lie in the relative interior of the set of all language independent communication strategies that induce it.

The rest of the paper is organized as follows. In Subsection 1.1 we discuss related literature. In Section 2 we provide preliminary definitions and results. We then characterize sets of belief vectors that can be a subset of the support of an inducible distribution over posterior belief vectors in Section 3. In Section 4 we introduce minimal and direct communication strategies and in Section 5 we turn to language independent communication strategies. In Section 6 we characterize inducible distributions of posteriors and provide several implications. Section 7 introduces information and posterior correspondences and in Section 8 we explore the informativeness of communication strategies.

\subsection{Related Literature}

Regarding the part of the paper where we focus on inducible distributions of posteriors, the closest study to ours is Arieli et al. (2020). They consider multiple receivers who share a common prior belief on a binary state space and study joint posterior belief distributions. They first show that for the case of two receivers a quantitative version of the Agreement Theorem of Aumann (1976) holds; beliefs of receivers are approximately equal when they are approximately common knowledge. For more than two receivers, they relate the feasibility condition to the No Trade Theorem of Milgrom and Stokey (1982) and provide a characterization of feasible joint posteriors. These characterizations are then applied to study independent joint posterior belief distributions. While we pose the same question as Arieli et al. (2020), we obtain a completely different characterization while allowing for an arbitrary finite state space. 
Another related paper is Ziegler (2019), which also considers feasible joint posteriors and follows a similar approach to Arieli et al. (2020). While the author also provides a characterization of feasible joint posteriors, Arieli et al. (2020) show that the necessary and sufficient condition provided by Ziegler (2019) becomes insufficient if the support of the marginal distributions contains more than two points.

In the single receiver case, introducing heterogeneity may render Bayes plausibility insufficient for a distribution to be inducible. Alonso and Camara (2016) consider a single receiver who does not share a common prior with the sender, and show that an additional condition is required on top of Bayes plausibility. Beauchêne et al. (2019) also consider a single receiver, who is ambiguity averse, and a sender who may use an ambiguous communication device. In that case they are able to show that a modified version of Bayes plausibility holds.

When there are multiple receivers, if information is perfectly correlated then Bayes plausibility is still the only condition for inducibility since in this case all receivers have the same ex-post belief. The first part of Wang (2013) and Alonso and Câmara (2016) both consider public communication and are examples of such a situation.

There is a wide literature that focuses on informativeness in the sense of Blackwell (1953). ${ }^{2}$ Rick (2013) considers an informed sender and an uninformed receiver and shows that miscommunication expands the set of distributions of beliefs the sender expects to induce. Gentzkow and Kamenica (2016) consider multiple senders and a single receiver and show that the amount of revealed information increases with the number of senders. Ichihashi (2019) considers a single sender and receiver where a designer can restrict the most informative signal that the sender can generate and characterizes the information restriction that maximizes the receiver's payoff. While these papers compare the informativeness of different information structures by investigating the induced distributions of posteriors, we analyze informativeness according to the higher order knowledge a receiver has about the posterior history.

\section{Preliminaries and Notation}

Let $N=\{1, \ldots, n\}$ be the set of receivers and $\Omega$ a finite set of states of the world. For any set $X$ denote by $\Delta(X)$ the set of probability distributions over $X$ with finite support. For finite $X$ denote by $\Delta^{\mathrm{o}}(X)$ the set of probability distribution over $X$ with full support. We assume that sender and receivers share a common prior belief $\lambda^{0} \in \Delta^{\circ}(\Omega)$.

Let $S_{i}$ be a non-empty set of messages sender can send to receiver $i \in N$ and let $S=\prod_{i \in N} S_{i}$. The elements of $S$ are called signals. A communication strategy is a function $\pi: \Omega \rightarrow \Delta(S)$ that maps each $\omega \in \Omega$ to a finite probability distribution over $S$. The set

\footnotetext{
${ }^{2} \mathrm{Li}$ (2017) considers a different criterion and measures informativeness in the sense of Ganuza and Penalva (2010), i.e. that more informative signals lead to greater variability of conditional expectations.
} 
of possible signal realizations is denoted by $S^{\pi}=\{s \in S \mid \exists \omega \in \Omega: \pi(s \mid \omega)>0\}$. Note that receiver $i \in N$ knows the joint distributions $\pi(\cdot \mid \omega)$ for all $\omega \in \Omega$, but only observes his private message $s_{i}$ when signal $s$ realizes. Denote the set of all communication strategies by $\Pi$. For each $\pi \in \Pi, s_{i} \in S_{i}$, and $\omega \in \Omega$, let

$$
\pi_{i}\left(s_{i} \mid \omega\right)=\sum_{t \in S: t_{i}=s_{i}} \pi(t \mid \omega)
$$

which is the probability that receiver $i \in N$ observes $s_{i}$ given that the true state is $\omega$. For each $i \in N$, define $S_{i}^{\pi}=\left\{s_{i} \in S_{i} \mid \exists \omega \in \Omega: \pi_{i}\left(s_{i} \mid \omega\right)>0\right\}$, which is the set of messages receiver $i$ observes with positive probability under $\pi$.

Given a communication strategy $\pi \in \Pi$, a signal $s \in S^{\pi}$ generates the posterior belief vector $\lambda^{s} \in \Delta(\Omega)^{n}$ defined by

$$
\lambda_{i}^{s}(\omega)=\frac{\pi_{i}\left(s_{i} \mid \omega\right) \lambda^{0}(\omega)}{\sum_{\omega^{\prime} \in \Omega} \pi_{i}\left(s_{i} \mid \omega^{\prime}\right) \lambda^{0}\left(\omega^{\prime}\right)}, \quad i \in N, \omega \in \Omega .
$$

So, $\lambda_{i}^{s}(\omega)$ is $i$ 's posterior belief that the true state is $\omega$ upon receiving message $s_{i}$.

A communication strategy $\pi \in \Pi$ induces the distribution $\sigma \in \Delta\left(\Delta(\Omega)^{n}\right)$ over posterior belief vectors if for all $\lambda \in \Delta(\Omega)^{n}$ it holds that

$$
\sigma(\lambda)=\sum_{s \in S^{\pi}: \lambda^{s}=\lambda} \sum_{\omega \in \Omega} \pi(s \mid \omega) \lambda^{0}(\omega) .
$$

In words, $\sigma(\lambda)$ is the probability of posterior belief vector $\lambda$. The distribution over posterior belief vectors induced by $\pi$ is denoted by $\sigma^{\pi}$. We define the set of inducible distributions over posterior belief vectors by

$$
\Sigma=\left\{\sigma \in \Delta\left(\Delta(\Omega)^{n}\right) \mid \exists \pi \in \Pi \text { such that } \sigma^{\pi}=\sigma\right\} .
$$

Observe that $\Sigma$ depends on the set $S$ of signals that the sender can use: a distribution $\sigma$ might only be inducible if $S$ is sufficiently rich. We will provide necessary and sufficient conditions on $S$ whenever appropriate.

Let $\sigma \in \Delta\left(\Delta(\Omega)^{n}\right)$ and denote the support of $\sigma$ by $R(\sigma)$. By our assumptions made so far, the support of $\sigma$ is a finite set. For each $i \in N$ and $\lambda_{i} \in \Delta(\Omega)$, define

$$
\sigma_{i}\left(\lambda_{i}\right)=\sum_{\lambda^{\prime} \in R(\sigma): \lambda_{i}^{\prime}=\lambda_{i}} \sigma\left(\lambda^{\prime}\right)
$$

That is, $\sigma_{i}\left(\lambda_{i}\right)$ is the probability that receiver $i$ has posterior belief $\lambda_{i}$. We denote the support of $\sigma_{i}$ by $R\left(\sigma_{i}\right)$. 
Let $\sigma, \sigma^{\prime} \in \Delta\left(\Delta(\Omega)^{n}\right)$ be two distributions over posterior belief vectors and let $\alpha \in$ $[0,1]$. The convex combination $\hat{\sigma}=\alpha \sigma+(1-\alpha) \sigma^{\prime}$ is defined by

$$
\hat{\sigma}(\lambda)=\alpha \sigma(\lambda)+(1-\alpha) \sigma^{\prime}(\lambda), \quad \lambda \in \Delta(\Omega)^{n} .
$$

Even in the case with a single receiver, $\Sigma$ need not be convex. For instance, if $S$ consists of two messages, then it is possible to induce $\sigma, \sigma^{\prime} \in \Sigma$ with disjoint supports of cardinality 2. If $\hat{\sigma}$ is a strictly convex combination of $\sigma$ and $\sigma^{\prime}$, then the cardinality of the support of $\hat{\sigma}$ equals 4 , which cannot be induced with two messages only. The next result shows that $\Sigma$ is convex when the signal space is sufficiently rich.

Proposition 2.1. Let $\sigma, \sigma^{\prime} \in \Sigma$ and $\alpha \in(0,1)$. Then $\alpha \sigma+(1-\alpha) \sigma^{\prime} \in \Sigma$ if and only if $\left|S_{i}\right| \geq\left|R\left(\sigma_{i}\right) \cup R\left(\sigma_{i}^{\prime}\right)\right|$ for all $i \in N$.

Proof. Let $\hat{\sigma}=\alpha \sigma+(1-\alpha) \sigma^{\prime}$.

If there is $i \in N$ such that $\left|S_{i}\right|<\left|R\left(\sigma_{i}\right) \cup R\left(\sigma_{i}^{\prime}\right)\right|$ then there are not sufficient messages to implement all of $i$ 's possible beliefs in $R\left(\hat{\sigma}_{i}\right)$.

For the other direction, let $\left|S_{i}\right| \geq\left|R\left(\sigma_{i}\right) \cup R\left(\sigma_{i}^{\prime}\right)\right|$ for all $i \in N$. Let $\pi, \pi^{\prime} \in \Pi$ be such that $\sigma^{\pi}=\sigma$ and $\sigma^{\pi^{\prime}}=\sigma^{\prime}$. Since $\left|S_{i}\right| \geq\left|R\left(\sigma_{i}\right) \cup R\left(\sigma_{i}^{\prime}\right)\right|$, we can assume without loss of generality that there is $s \in S$ with $s_{i} \in S_{i}^{\pi} \cap S_{i}^{\pi^{\prime}}$ if and only if there are $\lambda \in R(\sigma)$ and $\lambda^{\prime} \in R\left(\sigma^{\prime}\right)$ such that $\lambda_{i}=\lambda_{i}^{\prime}=\lambda_{i}^{s}$.

Let $\hat{\pi}=\alpha \pi+(1-\alpha) \pi^{\prime}$. Let $s \in S^{\hat{\pi}}$ and $i \in N$. Without loss of generality let $s_{i} \in S_{i}^{\pi}$. Assume first that $s_{i} \notin S_{i}^{\pi^{\prime}}$. It holds that, for every $\omega \in \Omega$,

$$
\hat{\lambda}_{i}^{s}(\omega)=\frac{\hat{\pi}_{i}\left(s_{i} \mid \omega\right) \lambda^{0}(\omega)}{\sum_{\omega^{\prime} \in \Omega} \hat{\pi}_{i}\left(s_{i} \mid \omega^{\prime}\right) \lambda^{0}\left(\omega^{\prime}\right)}=\frac{\alpha \pi_{i}\left(s_{i} \mid \omega\right) \lambda^{0}(\omega)}{\alpha \sum_{\omega^{\prime} \in \Omega} \pi_{i}\left(s_{i} \mid \omega^{\prime}\right) \lambda^{0}\left(\omega^{\prime}\right)}=\lambda_{i}^{s}(\omega) .
$$

Assume next that $s_{i} \in S_{i}^{\pi^{\prime}}$ and observe that in this case

$$
\frac{\pi_{i}\left(s_{i} \mid \omega\right) \lambda^{0}(\omega)}{\sum_{\omega^{\prime} \in \Omega} \pi_{i}\left(s_{i} \mid \omega^{\prime}\right) \lambda^{0}\left(\omega^{\prime}\right)}=\frac{\pi_{i}^{\prime}\left(s_{i} \mid \omega\right) \lambda^{0}(\omega)}{\sum_{\omega^{\prime} \in \Omega} \pi_{i}^{\prime}\left(s_{i} \mid \omega^{\prime}\right) \lambda^{0}\left(\omega^{\prime}\right)} .
$$

Thus,

$$
\hat{\lambda}_{i}^{s}(\omega)=\frac{\alpha \pi_{i}\left(s_{i} \mid \omega\right) \lambda^{0}(\omega)+(1-\alpha) \pi_{i}^{\prime}\left(s_{i} \mid \omega\right) \lambda^{0}(\omega)}{\alpha \sum_{\omega^{\prime} \in \Omega} \pi_{i}\left(s_{i} \mid \omega^{\prime}\right) \lambda^{0}\left(\omega^{\prime}\right)+(1-\alpha) \sum_{\omega^{\prime} \in \Omega} \pi_{i}^{\prime}\left(s_{i} \mid \omega^{\prime}\right) \lambda^{0}\left(\omega^{\prime}\right)}=\lambda_{i}^{s}(\omega) .
$$

We have shown that $R(\hat{\sigma})=R(\sigma) \cup R\left(\sigma^{\prime}\right)$. We now have, for every $\lambda \in \Delta(\Omega)^{n}$,

$$
\begin{aligned}
\hat{\sigma}(\lambda) & =\sum_{s \in S^{\hat{\pi}}: \hat{\lambda}^{s}=\lambda} \sum_{\omega \in \Omega} \hat{\pi}(s \mid \omega) \lambda^{0}(\omega) \\
& =\alpha \sum_{s \in S^{\pi}: \lambda^{s}=\lambda} \sum_{\omega \in \Omega} \pi(s \mid \omega) \lambda^{0}(\omega)+(1-\alpha) \sum_{s \in S^{\pi^{\prime}}: \lambda^{\prime s}=\lambda} \sum_{\omega \in \Omega} \pi^{\prime}(s \mid \omega) \lambda^{0}(\omega) \\
& =\alpha \sigma(\lambda)+(1-\alpha) \sigma^{\prime}(\lambda) .
\end{aligned}
$$

Hence, $\hat{\pi}$ induces $\hat{\sigma}$. 
Most of the literature considers $S_{i}$ an arbitrary set that contains all messages that are necessary. The previous proposition implies that in this case the set of inducible posteriors is convex.

A distribution over posterior belief vectors $\sigma \in \Delta\left(\Delta(\Omega)^{n}\right)$ is Bayes plausible if

$$
\sum_{\lambda_{i} \in R\left(\sigma_{i}\right)} \lambda_{i}(\omega) \sigma_{i}\left(\lambda_{i}\right)=\lambda^{0}(\omega), \quad i \in N, \omega \in \Omega .
$$

That is, for each receiver the expected posterior belief equals his prior belief. Kamenica and Gentzkow (2011) show that $\Sigma$ is the set of Bayes plausible posterior distributions in the single receiver case. It now follows for the multiple receiver case that every $\sigma \in \Sigma$ satisfies Bayes plausibility. We therefore obtain the following result, which is stated for later reference and without proof.

Proposition 2.2. Every $\sigma \in \Sigma$ is Bayes plausible.

\section{$3 \quad$ Implementing Belief Vectors}

When a sender is interacting with a single receiver who has no private information, Bayes plausibility of a distribution $\sigma \in \Delta\left(\Delta(\Omega)^{n}\right)$ is necessary and sufficient for $\sigma$ to belong to $\Sigma$. In particular, for any $\lambda \in \Delta(\Omega)$ there is $\sigma \in \Sigma$ such that $\sigma(\lambda)>0$. In contrast, in the multiple receiver case it is not true that any single posterior belief vector $\lambda \in \Delta(\Omega)^{n}$ can occur with positive probability for a suitably chosen communication strategy. Our first proposition shows that $\lambda \in \Delta(\Omega)^{n}$ can belong to the support of some $\sigma \in \Sigma$ if and only if there is at least one state which, according to $\lambda$, is deemed possible by all receivers.

Proposition 3.1. For every $i \in N$, let $S_{i}$ contain at least two messages. Let $\lambda \in \Delta(\Omega)^{n}$. There exists $\sigma \in \Sigma$ with $\sigma(\lambda)>0$ if and only if there is $\omega \in \Omega$ such that $\prod_{i \in N} \lambda_{i}(\omega)>0$.

Proof. Assume $\pi \in \Pi$ is such that $\sigma^{\pi}=\sigma$ with $\sigma(\lambda)>0$. Suppose that $\prod_{i \in N} \lambda_{i}(\omega)=0$ for all $\omega \in \Omega$, that is, for all $\omega \in \Omega$ there exists $i_{\omega} \in N$ such that $\lambda_{i_{\omega}}(\omega)=0$. Let $s \in S^{\pi}$ be such that $\lambda^{s}=\lambda$. Then it holds that, for all $\omega \in \Omega, \pi(s \mid \omega) \leq \pi_{i_{\omega}}\left(s_{i_{\omega}} \mid \omega\right)=0$. We find by (2) that $\sigma(\lambda)=0$, leading to a contradiction. Consequently, there exists $\omega \in \Omega$ such that $\prod_{i \in N} \lambda_{i}(\omega)>0$.

For the converse, assume there exists $\omega \in \Omega$ such that $\prod_{i \in N} \lambda_{i}(\omega)>0$. Let $i \in N$ and $\beta_{i}=\max _{\omega \in \Omega}\left(\lambda_{i}(\omega) / \lambda^{0}(\omega)\right)$ be the highest ratio across states of posterior belief to prior belief for receiver $i$. Let $x_{i}, y_{i} \in S_{i}$ be two distinct messages. We define, for every $\omega \in \Omega$,

$$
\begin{array}{rlrl}
\rho_{i}\left(x_{i} \mid \omega\right) & =\frac{1}{\beta_{i}} \frac{\lambda_{i}(\omega)}{\lambda^{0}(\omega)}, & \\
\rho_{i}\left(y_{i} \mid \omega\right) & =1-\rho_{i}\left(x_{i} \mid \omega\right), & \\
\rho_{i}\left(s_{i} \mid \omega\right) & =0, & & s_{i} \in S_{i} \backslash\left\{x_{i}, y_{i}\right\} .
\end{array}
$$


Notice that $\rho_{i}\left(x_{i} \mid \omega\right) \leq 1$. We obtain $\pi: \Omega \rightarrow \Delta(S)$ by defining, for every $\omega \in \Omega$,

$$
\pi(s \mid \omega)=\prod_{i \in N} \rho_{i}\left(s_{i} \mid \omega\right), \quad s \in S .
$$

It holds that $\pi$ is a communication strategy with $\pi_{i}\left(s_{i} \mid \omega\right)=\rho_{i}\left(s_{i} \mid \omega\right)$ for every receiver $i \in N$.

Let $i \in N$. For every $s \in S^{\pi}$ with $s_{i}=x_{i}$ it holds that

$\lambda_{i}^{s}(\omega)=\frac{\pi_{i}\left(x_{i} \mid \omega\right) \lambda^{0}(\omega)}{\sum_{\omega^{\prime} \in \Omega} \pi_{i}\left(x_{i} \mid \omega^{\prime}\right) \lambda^{0}\left(\omega^{\prime}\right)}=\frac{\frac{1}{\beta_{i}} \frac{\lambda_{i}(\omega)}{\lambda^{0}(\omega)} \lambda^{0}(\omega)}{\frac{1}{\beta_{i}} \sum_{\omega^{\prime} \in \Omega} \frac{\lambda_{i}\left(\omega^{\prime}\right)}{\lambda^{0}\left(\omega^{\prime}\right)} \lambda^{0}\left(\omega^{\prime}\right)}=\frac{\lambda_{i}(\omega)}{\sum_{\omega^{\prime} \in \Omega} \lambda_{i}\left(\omega^{\prime}\right)}=\lambda_{i}(\omega), \quad \omega \in \Omega$.

We have that $\lambda^{\bar{x}}=\lambda$, where $\bar{x}=\left(x_{1}, \ldots, x_{n}\right)$.

Let $\omega \in \Omega$ be such that $\lambda_{i}(\omega)>0$. Then

$$
\sigma(\lambda) \geq \pi(\bar{x} \mid \omega) \lambda^{0}(\omega)=\lambda^{0}(\omega) \prod_{i \in N} \rho_{i}\left(s_{i} \mid \omega\right)>0,
$$

which implies that $\lambda \in R\left(\sigma^{\pi}\right)$.

Let there be two receivers and a binary state space, say $\Omega=\{X, Y\}$, as in our example in the introduction. It follows from Proposition 3.1 that a posterior belief vector $\lambda$ with $\lambda(X)=(0,1)$ cannot result with positive probability under any communication protocol since $\lambda_{1}(X) \lambda_{2}(X)=0$ and $\lambda_{1}(Y) \lambda_{2}(Y)=0$. At the same time, for each $\varepsilon>0$, the posterior belief vector $\lambda$ with $\lambda(X)=(\varepsilon, 1)$ can be obtained with positive probability.

We now generalize Proposition 3.1 from a single posterior belief vector to finite sets of posterior belief vectors.

Proposition 3.2. Let $R \subseteq \Delta(\Omega)^{n}$ be finite. For every $i \in N$, let $S_{i}$ contain at least $\left|R_{i}\right|+1$ messages, where $R_{i}=\left\{\lambda_{i} \in \Delta(\Omega) \mid \lambda \in R\right\}$. There exists $\sigma \in \Sigma$ with $R \subseteq R(\sigma)$ if and only if for each $\lambda \in R$ there exists $\omega \in \Omega$ such that $\prod_{i \in N} \lambda_{i}(\omega)>0$.

Proof. Proposition 3.1 implies necessity. For the other direction, let $R_{i}=\left\{\lambda_{i}^{1}, \ldots, \lambda_{i}^{m_{i}}\right\}$, let $\left\{x_{i}^{1}, \ldots, x_{i}^{m_{i}}, y_{i}\right\} \subseteq S_{i}$ be such that $x_{i}^{k} \neq x_{i}^{\ell}, y_{i}$ for all $k \neq \ell$ and all $i \in N$. Let $R=\left\{\lambda^{1}, \ldots, \lambda^{m}\right\}$ and define $\pi^{1}, \ldots, \pi^{m}$ as in the proof of Proposition 3.1, where, for all $i \in N$ and all $k=1, \ldots, m$ one has $\lambda^{k} \in R\left(\sigma^{\pi^{k}}\right)$ and $S_{i}^{\pi^{k}} \subseteq\left\{x_{i}^{k}, y_{i}\right\}$. Let $\alpha^{1}, \ldots, \alpha^{m}>0$ with $\sum_{k=1}^{m} \alpha^{k}=1$, and let $\sigma=\sum_{k=1}^{m} \alpha^{k} \sigma^{\pi^{k}}$. Since $\left|S_{i}\right| \geq m_{i}+1=\left|\bigcup_{k=1}^{m} R\left(\sigma_{i}^{\pi^{k}}\right)\right|$, iterative application of Proposition 2.1 implies that $\sigma \in \Sigma$. Moreover, by construction, $\sigma^{\pi}\left(\lambda^{k}\right)=\alpha^{k} \sigma^{\pi^{k}}\left(\lambda^{k}\right)>0$.

At this point we have identified sets that can be subsets of the support of an inducible distribution over posterior belief vectors. In Section 6 we characterize all inducible distributions over posterior belief vectors and the sets that can be the support of such distributions. 


\section{Minimal and Direct Communication Strategies}

A large part of the literature is interested in "straightforward" communication strategies (Kamenica and Gentzkow, 2011) that send recommendations to receivers about what action to take. In the present paper, we do not specify sets of feasible actions for receivers, so that sending recommendations has no meaning. Nevertheless, some communication strategies are easier to handle than others and this and the next section will introduce some important classes.

Given a communication strategy $\pi \in \Pi$ and $s, s^{\prime} \in S^{\pi}$ with $s \neq s^{\prime}$, it is possible that $\lambda^{s}=\lambda^{s^{\prime}}$. That is, two distinct signals can generate the same posterior belief vector. This motivates the following definition.

Definition 4.1. A communication strategy $\pi \in \Pi$ is minimal if $\left|S^{\pi}\right|=\left|R\left(\sigma^{\pi}\right)\right|$. The set of minimal communication strategies is denoted by $\Pi^{\mathrm{m}}$

Under a minimal communication strategy, different signals lead to different posterior belief vectors. We give an illustration of a minimal communication strategy in the following example.

Example 4.2. Let $N=\{1,2\}, \Omega=\{X, Y\}, S_{1}=\{v, w\}$, and $S_{2}=\{w, x, y\}$. Assume that agents have a common prior $\lambda^{0}(X)=1 / 2$. Let $\pi$ be given as follows:

\begin{tabular}{c|ccc}
$\pi$ & $(v, x)$ & $(v, y)$ & $(w, w)$ \\
\hline$X$ & $\frac{1}{2}$ & 0 & $\frac{1}{2}$ \\
$Y$ & 0 & $\frac{1}{2}$ & $\frac{1}{2}$
\end{tabular}.

We have $S^{\pi}=\{(v, x),(v, y),(w, w)\}$. Irrespective of the signal received, receiver 1 gathers no information about the state: he has posterior beliefs $\lambda_{1}^{(v, x)}(X)=\lambda_{1}^{(v, y)}(X)=$ $\lambda_{1}^{(w, w)}(X)=1 / 2$. For receiver 2 , we have $\lambda_{2}^{(v, x)}(X)=1, \lambda_{2}^{(v, y)}(X)=0$, and $\lambda_{2}^{(w, w)}(X)=$ $1 / 2$. It follows that

$$
R\left(\sigma^{\pi}\right)=\{((1 / 2,1 / 2),(1,0)),((1 / 2,1 / 2),(0,1)),((1 / 2,1 / 2),(1 / 2,1 / 2))\} .
$$

Since $\left|S^{\pi}\right|=\left|R\left(\sigma^{\pi}\right)\right|, \pi$ is minimal.

In case of a single receiver, it is sufficient to have a bijection between $S^{\pi}$ and $R(\sigma)$ to ensure that each message leads to a different posterior, that is, to ensure that the communication strategy employs a minimal number of messages. If there are multiple receivers, however, the existence of such a bijection does not guarantee that the number of messages for each receiver is in fact minimal. For instance, the two messages $v, w$ in Example 4.1 both lead to the posterior belief $\lambda_{1}(X)=1 / 2$ for receiver 1 .

Definition 4.3. A communication strategy $\pi \in \Pi$ is direct if for all $i \in N$ it holds that $\left|S_{i}^{\pi}\right|=\left|R\left(\sigma_{i}^{\pi}\right)\right|$. The set of direct communication strategies is denoted by $\Pi^{\mathrm{d}}$. 
In other words, under a direct communication strategy any two different messages must lead to two different posterior beliefs. Hence, the number of different posterior beliefs a receiver can have equals the cardinality of $S_{i}^{\pi}$.

Example 4.4. Recall the minimal communication strategy $\pi$ in Example 4.2. Receiver 1 has the same posterior belief after observing $v$ and observing $w$, i.e., $\lambda_{1}^{(v, x)}(X)=\lambda_{1}^{(w, w)}(X)$. Thus, $\pi$ is not direct. Consider the communication strategy $\pi^{\prime}$ defined by:

\begin{tabular}{c|ccc}
$\pi^{\prime}$ & $(w, x)$ & $(w, y)$ & $(w, w)$ \\
\hline$X$ & $\frac{1}{2}$ & 0 & $\frac{1}{2}$ \\
$Y$ & 0 & $\frac{1}{2}$ & $\frac{1}{2}$
\end{tabular}.

We have $S^{\pi^{\prime}}=\{(w, x),(w, y),(w, w)\}$ and accordingly we can write the support of $\sigma^{\pi^{\prime}}$ as

$$
R\left(\sigma^{\pi^{\prime}}\right)=\{((1 / 2,1 / 2),(1,0)),((1 / 2,1 / 2),(0,1)),((1 / 2,1 / 2),(1 / 2,1 / 2))\} .
$$

Note that $R\left(\sigma^{\pi}\right)=R\left(\sigma^{\pi^{\prime}}\right)$. Since for all $s, t \in S^{\pi^{\prime}}$ and each $i \in N$ we have $\lambda_{i}^{\prime s}=\lambda_{i}^{\prime t}$ if and only if $s_{i}=t_{i}, \pi^{\prime}$ is direct.

For any communication strategy $\pi \in \Pi,\left|S_{i}^{\pi}\right|=\left|R\left(\sigma_{i}^{\pi}\right)\right|$ guarantees that a minimal number of messages is employed and implies that the number of employed signals is minimal as well. Thus, the following lemma does not come as a surprise.

Lemma 4.5. It holds that $\Pi^{\mathrm{d}} \subseteq \Pi^{\mathrm{m}}$.

Proof. Let $\pi \in \Pi^{\mathrm{d}}$. For each $i \in N$ there exists a bijection $\phi_{i}: S_{i}^{\pi} \rightarrow R\left(\sigma_{i}^{\pi}\right)$ since $\pi$ is direct. In particular, for every $s \in S^{\pi}$, we have $\lambda^{s}=\left(\phi_{i}\left(s_{i}\right)\right)_{i \in N}$ so that there is a bijection between $S^{\pi}$ and $R\left(\sigma^{\pi}\right)$. Hence, $\left|S^{\pi}\right|=\left|R\left(\sigma^{\pi}\right)\right|$, that is, $\pi$ is minimal.

We close this section by showing that any distribution in $\Sigma$ can be induced by a direct communication strategy. We do not provide a proof of Theorem 4.6 here, as it will follow easily from later results. The proof can be found after Corollary 6.3.

Theorem 4.6. If $\sigma \in \Sigma$, then there exists $\pi \in \Pi^{\mathrm{d}}$ such that $\sigma^{\pi}=\sigma$.

\section{$5 \quad$ Language Independent Communication Strategies}

The same distribution over posterior belief vectors can be induced by various communication strategies with potentially disjoint signal spaces. We now proceed to show that there is a canonical way to describe communication strategies. The principal idea is that the sender sends to each receiver the belief that he should have after observing the message.

Definition 5.1. A communication strategy $\pi \in \Pi$ is a language independent communication strategy (LICS) if $S^{\pi} \subseteq \Delta(\Omega)^{n}$ and, for all $s \in S^{\pi}, \lambda^{s}=s$. The set of language independent communication strategies is denoted by $\Pi^{\ell}$. 
Example 5.2. Let $N=\{1,2\}, \Omega=\{X, Y\}$, and $\lambda^{0}(X)=1 / 3$. The communication strategy $\pi \in \Pi$ is defined as follows:

\begin{tabular}{c|cccc}
$\pi$ & $(x, x)$ & $(x, y)$ & $(y, x)$ & $(y, y)$ \\
\hline$X$ & $\frac{1}{4}$ & $\frac{1}{4}$ & $\frac{1}{4}$ & $\frac{1}{4}$ \\
$Y$ & $\frac{1}{8}$ & $\frac{1}{8}$ & $\frac{1}{8}$ & $\frac{5}{8}$
\end{tabular}.

For any $i \in N$, we have $\lambda_{i}^{(x, x)}(X)=1 / 2$ and $\lambda_{i}^{(y, y)}(X)=1 / 4$. Hence, $\pi$ is in fact direct. The support of $\sigma^{\pi}$ is equal to

$$
\begin{aligned}
R\left(\sigma^{\pi}\right) & =\left\{\lambda^{(x, x)}, \lambda^{(x, y)}, \lambda^{(y, x)}, \lambda^{(y, y)}\right\} \\
& =\left\{\left(\left(\frac{1}{2}, \frac{1}{2}\right),\left(\frac{1}{2}, \frac{1}{2}\right)\right)\left(\left(\frac{1}{2}, \frac{1}{2}\right),\left(\frac{1}{4}, \frac{3}{4}\right)\right),\left(\left(\frac{1}{4}, \frac{3}{4}\right),\left(\frac{1}{2}, \frac{1}{2}\right)\right),\left(\left(\frac{1}{4}, \frac{3}{4}\right),\left(\frac{1}{4}, \frac{3}{4}\right)\right)\right\} .
\end{aligned}
$$

It holds that $\sigma^{\pi}\left(\lambda^{(x, x)}\right)=\sigma^{\pi}\left(\lambda^{(x, y)}\right)=\sigma^{\pi}\left(\lambda^{(y, x)}\right)=1 / 6$ and $\sigma^{\pi}\left(\lambda^{(y, y)}\right)=1 / 2$.

The communication strategy $\pi^{\prime} \in \Pi$ is obtained by switching messages $x$ and $y$, so

\begin{tabular}{c|cccc}
$\pi^{\prime}$ & $(x, x)$ & $(x, y)$ & $(y, x)$ & $(y, y)$ \\
\hline$X$ & $\frac{1}{4}$ & $\frac{1}{4}$ & $\frac{1}{4}$ & $\frac{1}{4}$ \\
$Y$ & $\frac{5}{8}$ & $\frac{1}{8}$ & $\frac{1}{8}$ & $\frac{1}{8}$
\end{tabular}.

It is immediate that $\sigma^{\pi}=\sigma^{\pi^{\prime}}$.

Next, consider the communication strategy $\hat{\pi}$ that corresponds to the convex combination of $\pi$ and $\pi^{\prime}$ with equal weights: $\hat{\pi}=1 / 2 \pi+1 / 2 \pi^{\prime}$. We have that

\begin{tabular}{c|cccc}
$\hat{\pi}$ & $(x, x)$ & $(x, y)$ & $(y, x)$ & $(y, y)$ \\
\hline$X$ & $\frac{1}{4}$ & $\frac{1}{4}$ & $\frac{1}{4}$ & $\frac{1}{4}$ \\
$Y$ & $\frac{3}{8}$ & $\frac{1}{8}$ & $\frac{1}{8}$ & $\frac{3}{8}$
\end{tabular}.

Perhaps surprisingly, it holds that $\sigma^{\hat{\pi}} \neq \sigma^{\pi}=\sigma^{\pi^{\prime}}$. It is easily verified that $\sigma^{\hat{\pi}}$ is the distribution that assigns probability 1 to the posterior belief vector $\left(\lambda^{0}, \lambda^{0}\right)$. It follows that the set of communication strategies which induce a particular distribution is not convex. Observe that $\hat{\pi}$ is not direct, which implies that $\Pi^{\mathrm{d}}$ is also not convex.

The communication strategies $\pi^{\ell}, \pi^{\prime \ell}$, and $\hat{\pi}^{\ell}$ are obtained by relabeling the signals sent by $\pi, \pi^{\prime}$, and $\hat{\pi}$, respectively, with the posterior belief vectors they lead to. We have that $\pi^{\ell}=\pi^{\prime \ell}$. Both are equal to

\begin{tabular}{c|cccc}
$\pi^{\ell}, \pi^{\prime \ell}$ & $\left(\left(\frac{1}{2}, \frac{1}{2}\right),\left(\frac{1}{2}, \frac{1}{2}\right)\right)$ & $\left(\left(\frac{1}{2}, \frac{1}{2}\right),\left(\frac{1}{4}, \frac{3}{4}\right)\right)$ & $\left(\left(\frac{1}{4}, \frac{3}{4}\right),\left(\frac{1}{2}, \frac{1}{2}\right)\right)$ & $\left(\left(\frac{1}{4}, \frac{3}{4}\right),\left(\frac{1}{4}, \frac{3}{4}\right)\right)$ \\
\hline$X$ & $\frac{1}{4}$ & $\frac{1}{4}$ & $\frac{1}{4}$ & $\frac{1}{4}$ \\
$Y$ & $\frac{1}{8}$ & $\frac{1}{8}$ & $\frac{1}{8}$ & $\frac{5}{8}$
\end{tabular}.

Each receiver has posterior belief $(1 / 2,1 / 2)$ upon observing message $(1 / 2,1 / 2)$ and has posterior belief $(1 / 4,3 / 4)$ upon observing message $(1 / 4,3 / 4)$. Thus, $\pi^{\ell}$ and $\pi^{\prime \ell}$ are language independent communication strategies.

Finally, $\hat{\pi}^{\ell}$ sends $\lambda^{0}$ to both players with probability 1 . 
The next result states that an LICS is direct.

Lemma 5.3. It holds that $\Pi^{\ell} \subseteq \Pi^{\mathrm{d}}$.

Proof. Let $\pi \in \Pi^{\ell}, s \in S^{\pi}$, and $i \in N$. It holds that $\lambda_{i}^{s}=s_{i}$ by definition of an LICS. This defines an identity between $S_{i}^{\pi}$ and $R\left(\sigma_{i}^{\pi}\right)$. It follows that $\left|S_{i}^{\pi}\right|=\left|R\left(\sigma_{i}^{\pi}\right)\right|$.

By Lemma 5.3 we know that an LICS is direct and by Lemma 4.5 directness implies minimality. Thus, there is a chain of inclusions between $\Pi^{\ell}, \Pi^{\mathrm{d}}$, and $\Pi^{\mathrm{m}}$.

Corollary 5.4. It holds that $\Pi^{\ell} \subseteq \Pi^{\mathrm{d}} \subseteq \Pi^{\mathrm{m}} \subseteq \Pi$.

Since we can transform any given direct communication strategy into an LICS by relabeling each message with the posterior belief that message leads to, an immediate consequence of Theorem 4.6 is that any element of $\Sigma$ can be induced by an LICS if $\Delta(\Omega)^{n} \subseteq S$, a result also obtained by Arieli et al. (2020) for a binary state space. One advantage of language independent communication strategies is that for each $\sigma \in \Sigma$ the set of all language independent communication strategies that induce $\sigma$, denoted by $\Pi^{\ell}(\sigma)$, is convex. The proof of this statement, however, is postponed as it follows easily from later results. The proof can be found after Corollary 6.3.

Proposition 5.5. Let $\Delta(\Omega)^{n} \subseteq S$ and $\sigma \in \Sigma$. Then $\Pi^{\ell}(\sigma)$ is non-empty and convex.

It follows from Example 5.2 that both the set of all communication strategies and the set of all direct communication strategies that induce a given $\sigma$ is typically not convex.

Recall that given a direct communication strategy, we can obtain an LICS by simply replacing messages with the posterior beliefs they lead to. More generally, given a communication strategy $\pi \in \Pi$, one can define $\pi^{\prime} \in \Pi$ by a one-to-one change in the names of messages in $S_{i}^{\pi}$ for each $i \in N$. In this case, we typically have $S^{\pi^{\prime}} \neq S^{\pi}$, though we intuitively think of both communication strategies as equivalent. More formally, we have the following definition.

Definition 5.6. Two communication strategies $\pi: \Omega \rightarrow \Delta(S)$ and $\hat{\pi}: \Omega \rightarrow \Delta(\hat{S})$ are equivalent $(\pi \sim \hat{\pi})$ if for every $i \in N$ there is a bijection $\psi_{i}: S_{i}^{\pi} \rightarrow \hat{S}_{i}^{\hat{\pi}}$ such that, for every $\omega \in \Omega$, for every $s \in S^{\pi}, \hat{\pi}(\psi(s) \mid \omega)=\pi(s \mid \omega)$.

We can interpret equivalent communication strategies as providing the same information in different languages. Indeed, let $s_{i} \in S_{i}^{\pi}$ and $\hat{s}_{i} \in \hat{S}_{i}^{\hat{\pi}}$ be such that $\psi_{i}\left(s_{i}\right)=\hat{s}_{i}$. It holds that

$$
\pi_{i}\left(s_{i} \mid \omega\right)=\sum_{t \in S^{\pi}: t_{i}=s_{i}} \pi(t \mid \omega)=\sum_{t \in S^{\pi}: t_{i}=s_{i}} \hat{\pi}(\psi(t) \mid \omega)=\sum_{\hat{t} \in \hat{S}^{\hat{\pi}}: \hat{t}_{i}=\hat{s}_{i}} \hat{\pi}(\hat{t} \mid \omega)=\hat{\pi}_{i}\left(\hat{s}_{i} \mid \omega\right), \quad \omega \in \Omega .
$$


Now consider $s \in S^{\pi}$ and $\hat{s} \in \hat{S}^{\hat{\pi}}$ such that $\hat{s}=\psi(s)$. For every $i \in N$, we have that

$$
\lambda_{i}^{s}(\omega)=\frac{\pi_{i}\left(s_{i} \mid \omega\right) \lambda^{0}(\omega)}{\sum_{\omega^{\prime} \in \Omega} \pi_{i}\left(s_{i} \mid \omega^{\prime}\right) \lambda^{0}\left(\omega^{\prime}\right)}=\frac{\hat{\pi}_{i}\left(\hat{s}_{i} \mid \omega\right) \lambda^{0}(\omega)}{\sum_{\omega^{\prime} \in \Omega} \hat{\pi}_{i}\left(\hat{s}_{i} \mid \omega^{\prime}\right) \lambda^{0}\left(\omega^{\prime}\right)}=\hat{\lambda}_{i}^{\hat{s}}(\omega) .
$$

It follows from (5) that sending signal $s$ under communication strategy $\pi$ and sending signal $\hat{s}$ under communication strategy $\hat{\pi}$ results in the same posterior belief vector. It is also immediate from Definition 5.6 that $\hat{S}^{\hat{\pi}}=\psi\left(S^{\pi}\right)$.

The next proposition, stating that equivalent communication strategies induce the same distribution over posterior belief vectors, now follows easily.

Proposition 5.7. Let $\pi: \Omega \rightarrow \Delta(S)$ and $\hat{\pi}: \Omega \rightarrow \Delta(\hat{S})$ be such that $\pi \sim \hat{\pi}$. It holds that $\sigma^{\pi}=\sigma^{\hat{\pi}}$.

Proof. For every $i \in N$ there is a bijection $\psi_{i}: S_{i}^{\pi} \rightarrow \hat{S}_{i}^{\hat{\pi}}$ such that, for every $\omega \in \Omega$, for every $s \in S^{\pi}, \hat{\pi}(\psi(s) \mid \omega)=\pi(s \mid \omega)$. Let $s \in S^{\pi}$ and $\hat{s} \in \hat{S}^{\hat{\pi}}$ be such that $\psi(s)=\hat{s}$. It follows from (5) that $\lambda^{s}=\hat{\lambda}^{\hat{s}}$. Since $\hat{S}^{\hat{\pi}}=\psi\left(S^{\pi}\right)$, we have that $R\left(\sigma^{\hat{\pi}}\right)=R\left(\sigma^{\pi}\right)$. Moreover, it holds that, for every $\lambda \in R\left(\sigma^{\pi}\right)$,

$$
\begin{aligned}
\sigma^{\pi}(\lambda) & =\sum_{s \in S^{\pi}: \lambda^{s}=\lambda} \sum_{\omega \in \Omega} \pi(s \mid \omega) \lambda^{0}(\omega)=\sum_{s \in S^{\pi}: \lambda^{s}=\lambda} \sum_{\omega \in \Omega} \hat{\pi}(\psi(s) \mid \omega) \lambda^{0}(\omega) \\
& =\sum_{\hat{s} \in \hat{S}^{\hat{\pi}}: \hat{\lambda}^{\hat{s}}=\lambda} \sum_{\omega \in \Omega} \hat{\pi}(\hat{s} \mid \omega) \lambda^{0}(\omega)=\sigma^{\hat{\pi}}(\lambda) .
\end{aligned}
$$

Note that the converse of Proposition 5.7 is not true: as we will see in Example 6.6 there are communication strategies that induce the same distribution over posterior belief vectors but that are not equivalent.

The next proposition and corollary makes clear that each set of equivalent communication strategies contains at most one LICS.

Proposition 5.8. Let $\pi, \pi^{\prime} \in \Pi^{\ell}$ with $\pi \sim \pi^{\prime}$. It holds that $\pi=\pi^{\prime}$.

Proof. By Proposition 5.7 it holds that $\sigma^{\pi}=\sigma^{\pi^{\prime}}$, so $S^{\pi}=R\left(\sigma^{\pi}\right)=R\left(\sigma^{\pi^{\prime}}\right)=S^{\pi^{\prime}}$. As $\pi \sim \pi^{\prime}$, for every $i \in N$ there is a bijection $\psi_{i}: S_{i}^{\pi} \rightarrow S_{i}^{\pi^{\prime}}$ such that, for every $\omega \in \Omega$, for every $s \in S^{\pi}, \pi^{\prime}(\psi(s) \mid \omega)=\pi(s \mid \omega)$. In particular, since $\pi, \pi^{\prime} \in \Pi^{\ell}$, we have, for every $i \in N$, for every $\lambda \in S^{\pi}$,

$$
\psi_{i}\left(\lambda_{i}\right)(\omega)=\frac{\pi_{i}^{\prime}\left(\psi_{i}\left(\lambda_{i}\right) \mid \omega\right) \lambda^{0}(\omega)}{\sum_{\omega^{\prime} \in \Omega} \pi_{i}^{\prime}\left(\psi_{i}\left(\lambda_{i}\right) \mid \omega^{\prime}\right) \lambda^{0}\left(\omega^{\prime}\right)}=\frac{\pi_{i}\left(\lambda_{i} \mid \omega\right) \lambda^{0}(\omega)}{\sum_{\omega^{\prime} \in \Omega} \pi_{i}\left(\lambda_{i} \mid \omega^{\prime}\right) \lambda^{0}\left(\omega^{\prime}\right)}=\lambda_{i}(\omega), \quad \omega \in \Omega,
$$

where the first and third equality follow since $\pi, \pi^{\prime} \in \Pi^{\ell}$ and the second equality uses (5). It follows that $\pi=\pi^{\prime}$. 
Observe that a communication strategy that is not direct cannot be equivalent to an LICS as the required bijection between message spaces cannot exist. Nevertheless for every communication strategy there is a canonical way to find an LICS that induces the same posterior. The construction heavily lies on the following lemma, which is straightforward and therefore stated without proof. ${ }^{3}$

Lemma 5.9. Let $\pi \in \Pi$ be a communication strategy. It holds that

$$
\frac{\sum_{s_{i} \in S_{i}^{\pi}: \lambda_{i}^{s}=\lambda_{i}} \pi_{i}\left(s_{i} \mid \omega\right) \lambda^{0}(\omega)}{\sum_{\omega^{\prime} \in \Omega} \sum_{s_{i} \in S_{i}^{\pi}: \lambda_{i}^{s}=\lambda_{i}} \pi_{i}\left(s_{i} \mid \omega^{\prime}\right) \lambda^{0}\left(\omega^{\prime}\right)}=\lambda_{i}(\omega), \quad \omega \in \Omega, i \in N, \lambda_{i} \in R\left(\sigma_{i}^{\pi}\right) .
$$

Lemma 5.9 extends the formula for Bayesian updating and applies it to all messages simultaneously that lead a particular posterior belief. According to the lemma, distinct messages that lead to the same posterior can be replaced by the same message. Thus, the following corollary is immediate.

Corollary 5.10. Let $\Delta(\Omega)^{n} \subseteq S$. For $\pi \in \Pi$ define $\pi^{\ell}: \Omega \rightarrow \Delta(S)$ as

$$
\pi^{\ell}(\lambda \mid \omega)=\sum_{s \in S^{\pi}: \lambda^{s}=\lambda} \pi(s \mid \omega), \quad \omega \in \Omega, \lambda \in R\left(\sigma^{\pi}\right)
$$

Then $\sigma^{\pi^{\ell}}=\sigma^{\pi}$. Moreover, if $\pi \in \Pi^{d}$ then $\pi^{\ell}$ is equivalent to $\pi$.

\section{Inducible Distributions}

Unlike the single receiver case, when dealing with multiple receivers Bayes plausibility alone is not sufficient to ensure that a distribution over posterior belief vectors belongs to $\Sigma$.

Example 6.1. Let $N=\{1,2,3\}, \Omega=\{X, Y\}$, and $S=\Delta(\Omega)^{3}$. Assume the agents have common prior $\lambda^{0}(X)=1 / 6$. Let $\lambda^{1}(X)=(1 / 2,1 / 2,0), \lambda^{2}(X)=(1 / 2,0,1 / 2)$, $\lambda^{3}(X)=(0,1 / 2,1 / 2)$, and $\lambda^{4}(X)=(0,0,0)$ and let $\sigma \in \Delta\left(\Delta(\Omega)^{3}\right)$ be given by $\sigma\left(\lambda^{1}\right)=$ $\sigma\left(\lambda^{2}\right)=\sigma\left(\lambda^{3}\right)=1 / 6$ and $\sigma\left(\lambda^{4}\right)=1 / 2$. Then, for each $i \in N$, we have $\sigma_{i}(1 / 2,1 / 2)=1 / 3$ and $\sigma_{i}(0,1)=2 / 3$.

First note that $\sigma$ is Bayes plausible:

$$
\sum_{\lambda_{i} \in R\left(\sigma_{i}\right)} \lambda_{i}(X) \sigma_{i}\left(\lambda_{i}\right)=\frac{1}{2} \cdot \sigma_{i}(1 / 2,1 / 2)+0 \cdot \sigma_{i}(0,1)=\frac{1}{2} \cdot \frac{1}{3}=\frac{1}{6}=\lambda^{0}(X), \quad i \in N .
$$

Suppose that communication strategy $\pi \in \Pi$ induces $\sigma$. By Corollary 5.10 it is without loss of generality to assume that $\pi \in \Pi^{\ell}$. In this case, for any receiver, observing $(1 / 2,1 / 2)$

\footnotetext{
${ }^{3}$ It is implied by the proof of Lemma 3.4 in Kerman et al. (2020).
} 
leads to posterior belief $(1 / 2,1 / 2)$ and observing $(0,1)$ leads to posterior belief $(0,1)$. This implies that receivers cannot observe $(0,1)$ in state $X$, i.e., $\pi_{i}((0,1) \mid X)=0$ for all $i \in N$. It follows that $\pi\left(\lambda^{1} \mid X\right)=\pi\left(\lambda^{2} \mid X\right)=\pi\left(\lambda^{3} \mid X\right)=\pi\left(\lambda^{4} \mid X\right)=0$, which obviously leads to a contradiction.

To guarantee that a distribution over posterior belief vectors belongs to $\Sigma$, additional conditions need to be imposed on top of Bayes plausibility. In Theorem 6.2, we provide necessary and sufficient conditions for a distribution over posterior belief vectors to belong to $\Sigma$.

Theorem 6.2. Let $\sigma \in \Delta\left(\Delta(\Omega)^{n}\right)$ be such that, for every $i \in N,\left|S_{i}\right| \geq\left|R\left(\sigma_{i}\right)\right|$. Then $\sigma \in \Sigma$ if and only if $\sigma$ is Bayes plausible and there exists $p \in \mathbb{R}_{+}^{\Omega \times R(\sigma)}$ such that

$$
\begin{array}{lll}
\text { (i) } & \sum_{\omega \in \Omega} p(\omega, \lambda)=\sigma(\lambda), & \lambda \in R(\sigma), \\
\text { (ii) } & \sum_{\lambda^{\prime} \in R(\sigma): \lambda_{i}^{\prime}=\lambda_{i}} p\left(\omega, \lambda^{\prime}\right)=\lambda_{i}(\omega) \sigma_{i}\left(\lambda_{i}\right), & \omega \in \Omega, i \in N, \lambda_{i} \in R\left(\sigma_{i}\right) .
\end{array}
$$

If $\sigma \in \Sigma$, then the communication strategy $\pi: \Omega \rightarrow \Delta\left(\Delta(\Omega)^{n}\right)$ defined by

$$
\pi(\lambda \mid \omega)=\frac{p(\omega, \lambda)}{\lambda^{0}(\omega)}, \quad \omega \in \Omega, \lambda \in R(\sigma)
$$

is an LICS such that $\sigma^{\pi}=\sigma$.

Proof. Assume that $\sigma$ is Bayes plausible and there exists $p \in \mathbb{R}_{+}^{\Omega \times R(\sigma)}$ such that $(i)$ and (ii) are satisfied. Let $\pi$ be defined as in (8). We first show that $\pi$ is a communication strategy.

Let $\omega \in \Omega$. Obviously, it holds that, for every $\lambda \in \Delta(\Omega)^{n}, \pi(\lambda \mid \omega) \geq 0$. In formula (9) that follows next, $i \in N$ is an arbitrarily chosen receiver. It holds that

$$
\sum_{\lambda \in S^{\pi}} p(\omega, \lambda)=\sum_{\lambda_{i} \in R_{i}\left(\sigma_{i}\right)} \sum_{\lambda^{\prime} \in R(\sigma): \lambda_{i}^{\prime}=\lambda_{i}} p\left(\omega, \lambda^{\prime}\right) \stackrel{(i i)}{=} \sum_{\lambda_{i} \in R_{i}\left(\sigma_{i}\right)} \lambda_{i}(\omega) \sigma_{i}\left(\lambda_{i}\right)=\lambda^{0}(\omega),
$$

where the last equality is true as $\sigma$ is Bayes plausible. We find that

$$
\sum_{\lambda \in S^{\pi}} \pi(\lambda \mid \omega)=\sum_{\lambda \in S^{\pi}} \frac{p(\omega, \lambda)}{\lambda^{0}(\omega)} \stackrel{(9)}{=} \frac{\lambda^{0}(\omega)}{\lambda^{0}(\omega)}=1
$$

which proves that $\pi$ is a communication strategy. 
Next, we show that $\pi$ is an LICS. Let $\omega \in \Omega, i \in N$, and $\lambda_{i} \in R\left(\sigma_{i}\right)$. It holds that

$$
\begin{aligned}
\frac{\pi_{i}\left(\lambda_{i} \mid \omega\right) \lambda^{0}(\omega)}{\sum_{\omega^{\prime} \in \Omega} \pi_{i}\left(\lambda_{i} \mid \omega^{\prime}\right) \lambda^{0}\left(\omega^{\prime}\right)} & =\frac{\sum_{\lambda^{\prime} \in R(\sigma): \lambda_{i}^{\prime}=\lambda_{i}} \pi\left(\lambda^{\prime} \mid \omega\right) \lambda^{0}(\omega)}{\sum_{\omega^{\prime} \in \Omega} \sum_{\lambda^{\prime} \in R(\sigma): \lambda_{i}^{\prime}=\lambda_{i}} \pi\left(\lambda^{\prime} \mid \omega^{\prime}\right) \lambda^{0}\left(\omega^{\prime}\right)} \\
& \stackrel{(8)}{=} \frac{\sum_{\lambda^{\prime} \in R(\sigma): \lambda_{i}^{\prime}=\lambda_{i}} \frac{p\left(\omega, \lambda^{\prime}\right)}{\lambda^{0}(\omega)} \lambda^{0}(\omega)}{\sum_{\omega^{\prime} \in \Omega} \sum_{\lambda^{\prime} \in R(\sigma): \lambda_{i}^{\prime}=\lambda_{i}} \frac{p\left(\omega^{\prime}, \lambda^{\prime}\right)}{\lambda^{0}\left(\omega^{\prime}\right)} \lambda^{0}\left(\omega^{\prime}\right)} \\
& =\frac{\sum_{\lambda^{\prime} \in R(\sigma): \lambda_{i}^{\prime}=\lambda_{i}} p\left(\omega, \lambda^{\prime}\right)}{\sum_{\omega^{\prime} \in \Omega} \sum_{\lambda^{\prime} \in R(\sigma): \lambda_{i}^{\prime}=\lambda_{i}} p\left(\omega^{\prime}, \lambda^{\prime}\right)} \\
& \stackrel{(i i)}{=} \frac{\lambda_{i}(\omega) \sigma_{i}\left(\lambda_{i}\right)}{\sum_{\lambda^{\prime} \in R(\sigma): \lambda_{i}^{\prime}=\lambda_{i}} \sum_{\omega^{\prime} \in \Omega} p\left(\omega^{\prime}, \lambda^{\prime}\right)} \\
& \stackrel{(i)}{=} \frac{\lambda_{i}(\omega) \sigma_{i}\left(\lambda_{i}\right)}{\sum_{\lambda^{\prime} \in R(\sigma): \lambda_{i}^{\prime}=\lambda_{i}} \sigma\left(\lambda^{\prime}\right)} \\
& =\frac{\lambda_{i}(\omega) \sigma_{i}\left(\lambda_{i}\right)}{\sigma_{i}\left(\lambda_{i}\right)} \\
& =\lambda_{i}(\omega) .
\end{aligned}
$$

As message $\lambda_{i}$ leads to posterior $\lambda_{i}, \pi$ is an LICS.

We show next that $\sigma^{\pi}=\sigma$. Let $\lambda \in R(\sigma)$. It holds that

$$
\sigma^{\pi}(\lambda)=\sum_{\omega \in \Omega} \pi(\lambda \mid \omega) \lambda^{0}(\omega)=\sum_{\omega \in \Omega} \frac{p(\omega, \lambda)}{\lambda^{0}(\omega)} \lambda^{0}(\omega)=\sum_{\omega \in \Omega} p(\omega, \lambda) \stackrel{(i)}{=} \sigma(\lambda) .
$$

At this point we have shown that $\sigma$ is inducible if $R\left(\sigma_{i}\right) \subseteq S_{i}$. Recall that $\left|S_{i}\right| \geq R\left(\sigma_{i}\right)$. For every $i \in N$, let $T_{i}$ be a subset of $S_{i}$ with cardinality equal to $\left|R\left(\sigma_{i}\right)\right|$ and take a bijection $\psi_{i}: R\left(\sigma_{i}\right) \rightarrow T_{i}$. The communication strategy $\pi^{\prime}: \Omega \rightarrow \Delta(S)$ is defined by

$$
\pi^{\prime}(\psi(\lambda) \mid \omega)=\pi(\lambda \mid \omega), \quad \omega \in \Omega, \lambda \in R(\sigma) .
$$

It holds that $\pi \sim \pi^{\prime}$, so by Proposition 5.7 we have that $\sigma^{\pi^{\prime}}=\sigma^{\pi}=\sigma$. It follows that $\sigma \in \Sigma$.

Now assume that $\sigma \in \Sigma$. It follows from Proposition 2.2 that $\sigma$ is Bayes plausible. Let $\pi \in \Pi$ be such that $\sigma^{\pi}=\sigma$. For every $\omega \in \Omega$, for every $\lambda \in R(\sigma)$, define

$$
p(\omega, \lambda)=\sum_{s \in S^{\pi}: \lambda^{s}=\lambda} \pi(s \mid \omega) \lambda^{0}(\omega)
$$

We first show that $(i)$ holds. We have that

$$
\sigma(\lambda)=\sum_{s \in S^{\pi}: \lambda^{s}=\lambda} \sum_{\omega \in \Omega} \pi(s \mid \omega) \lambda^{0}(\omega) \stackrel{(10)}{=} \sum_{\omega \in \Omega} p(\omega, \lambda), \quad \lambda \in R(\sigma) .
$$


Next, we show $(i i)$ holds. Let $\omega \in \Omega, i \in N$, and $\lambda_{i} \in R\left(\sigma_{i}\right)$. We have that

$$
\begin{aligned}
& \lambda_{i}(\omega) \sigma_{i}\left(\lambda_{i}\right)=\frac{\sum_{s_{i} \in S_{i}^{\pi}: \lambda_{i}^{s}=\lambda_{i}} \pi_{i}\left(s_{i} \mid \omega\right) \lambda^{0}(\omega)}{\sum_{\omega^{\prime} \in \Omega} \sum_{s_{i} \in S_{i}^{\pi}: \lambda_{i}^{s}=\lambda_{i}} \pi_{i}\left(s_{i} \mid \omega^{\prime}\right) \lambda^{0}\left(\omega^{\prime}\right)} \sum_{\lambda^{\prime} \in R(\sigma): \lambda_{i}^{\prime}=\lambda_{i}} \sigma\left(\lambda^{\prime}\right) \\
& =\frac{\sum_{s_{i} \in S_{i}^{\pi}: \lambda_{i}^{s}=\lambda_{i}} \pi_{i}\left(s_{i} \mid \omega\right) \lambda^{0}(\omega)}{\sum_{\omega^{\prime} \in \Omega} \sum_{s_{i} \in S_{i}^{\pi}: \lambda_{i}^{s}=\lambda_{i}} \pi_{i}\left(s_{i} \mid \omega^{\prime}\right) \lambda^{0}\left(\omega^{\prime}\right)} \sum_{\lambda^{\prime} \in R(\sigma): \lambda_{i}^{\prime}=\lambda_{i}} \sum_{s \in S^{\pi}: \lambda^{s}=\lambda^{\prime}} \sum_{\omega^{\prime} \in \Omega} \pi\left(s \mid \omega^{\prime}\right) \lambda^{0}\left(\omega^{\prime}\right) \\
& =\frac{\sum_{s_{i} \in S_{i}^{\pi}: \lambda_{i}^{s}=\lambda_{i}} \pi_{i}\left(s_{i} \mid \omega\right) \lambda^{0}(\omega)}{\sum_{\omega^{\prime} \in \Omega} \sum_{s_{i} \in S_{i}^{\pi}: \lambda_{i}^{s}=\lambda_{i}} \pi_{i}\left(s_{i} \mid \omega^{\prime}\right) \lambda^{0}\left(\omega^{\prime}\right)} \sum_{\omega^{\prime} \in \Omega} \sum_{s_{i} \in S_{i}^{\pi}: \lambda_{i}^{s}=\lambda_{i}} \pi_{i}\left(s_{i} \mid \omega^{\prime}\right) \lambda^{0}\left(\omega^{\prime}\right) \\
& =\sum_{s_{i} \in S_{i}^{\pi}: \lambda_{i}^{s}=\lambda_{i}} \pi_{i}\left(s_{i} \mid \omega\right) \lambda^{0}(\omega) \\
& =\sum_{\lambda^{\prime} \in R(\sigma): \lambda_{i}^{\prime}=\lambda_{i}} \sum_{s \in S^{\pi}: \lambda^{s}=\lambda^{\prime}} \pi(s \mid \omega) \lambda^{0}(\omega) \\
& =\sum_{\lambda^{\prime} \in R(\sigma): \lambda_{i}^{\prime}=\lambda_{i}} p\left(\omega, \lambda^{\prime}\right) \text {, }
\end{aligned}
$$

where the first equality follows from Lemma 5.9.

Theorem 6.2 makes explicit what is needed in addition to Bayes plausibility to ensure that a distribution over posterior belief vectors belongs to $\Sigma$. Observe that any $p \in \mathbb{R}_{+}^{\Omega \times R(\sigma)}$ which satisfies Condition $(i)$ is a probability distribution, that is, $p \in \Delta(\Omega \times R(\sigma))$. Condition $(i)$ can be interpreted as "posterior marginality" as it states that the probability of a posterior belief vector $\lambda$ is the marginal of $p(\omega, \lambda)$. The right-hand side of condition (ii) is the probability that $\omega$ is the true state according to $i$ 's belief $\lambda_{i}$ multiplied with the probability that $i$ has belief $\lambda_{i}$. Thus, the sum on the left-hand side is the probability that $i$ has belief $\lambda_{i}$ and $\omega$ is the true state.

For any $\sigma \in \Sigma$ define

$$
P(\sigma)=\left\{p \in \mathbb{R}_{+}^{\Omega \times R(\sigma)} \mid p \text { satisfies (i) and (ii) of Theorem 6.2 }\right\} .
$$

As $P(\sigma)$ is defined as the set of non-negative matrix solutions to a system of linear equalities, where the system is such that the components of any solution matrix sum up to one, we immediately have the following result.

Corollary 6.3. For every $\sigma \in \Sigma, P(\sigma)$ is a non-empty, compact, and convex polytope.

We are now ready to provide the remaining proofs of Sections 5 and 6 .

Proof of Theorem 4.6. Let $\sigma \in \Sigma$. Then it holds that, for every $i \in N,\left|S_{i}\right| \geq R\left(\sigma_{i}\right)$. Theorem 6.2 implies that there is an LICS $\pi: \Omega \rightarrow \Delta\left(\Delta(\Omega)^{n}\right)$ which induces $\sigma$. For every 
$i \in N$, let $T_{i}$ be a subset of $S_{i}$ with cardinality equal to $\left|R\left(\sigma_{i}\right)\right|$ and take a bijection $\psi_{i}: R\left(\sigma_{i}\right) \rightarrow T_{i}$. The communication strategy $\pi^{\prime}: \Omega \rightarrow \Delta(S)$ is defined by

$$
\pi^{\prime}(\psi(\lambda) \mid \omega)=\pi(\lambda \mid \omega), \quad \omega \in \Omega, \lambda \in R(\sigma) .
$$

It holds that $\pi \sim \pi^{\prime}$, so by Proposition 5.7 we have that $\sigma^{\pi^{\prime}}=\sigma^{\pi}=\sigma$. As the LICS $\pi$ is direct, it follows that $\pi^{\prime} \in \Pi^{\mathrm{d}}$.

Proof of Proposition 5.5. As $P(\sigma)$ is a non-empty, compact, and convex polytope by Corollary 6.3 and $\Pi^{\ell}(\sigma)$ is a linear transformation of $P(\sigma)$ by $(8), \Pi^{\ell}(\sigma)$ is a non-empty, compact, and convex polytope as well.

In the next example, we make use of Theorem 6.2 to determine whether a given distribution over posterior belief vectors belongs to $\Sigma$.

Example 6.4. Recall the distribution over posterior belief vectors $\sigma$ in Example 6.1 with

$$
\begin{aligned}
R(\sigma) & =\left\{\lambda^{1}, \lambda^{2}, \lambda^{3}, \lambda^{4}\right\} \\
& =\left\{\left(\left(\frac{1}{2}, \frac{1}{2}\right),\left(\frac{1}{2}, \frac{1}{2}\right),(0,1)\right),\left(\left(\frac{1}{2}, \frac{1}{2}\right),(0,1),\left(\frac{1}{2}, \frac{1}{2}\right)\right),\left((0,1),\left(\frac{1}{2}, \frac{1}{2}\right),\left(\frac{1}{2}, \frac{1}{2}\right)\right),((0,1),(0,1),(0,1))\right\} .
\end{aligned}
$$

Moreover, we have $\sigma\left(\lambda^{1}\right)=\sigma\left(\lambda^{2}\right)=\sigma\left(\lambda^{3}\right)=1 / 6$ and $\sigma\left(\lambda^{4}\right)=1 / 2$.

Suppose $\sigma \in \Sigma$. Then, by Theorem 6.2 there exists $p \in P(\sigma)$ such that

$$
\begin{aligned}
& p\left(X, \lambda^{1}\right)+p\left(X, \lambda^{2}\right)=p\left(X, \lambda^{1}\right)+p\left(X, \lambda^{3}\right)=p\left(X, \lambda^{2}\right)+p\left(X, \lambda^{3}\right)=\frac{1}{6} \\
& p\left(X, \lambda^{1}\right)+p\left(X, \lambda^{4}\right)=p\left(X, \lambda^{2}\right)+p\left(X, \lambda^{4}\right)=p\left(X, \lambda^{3}\right)+p\left(X, \lambda^{4}\right)=0
\end{aligned}
$$

where we make use of Condition (ii) for $\omega=X$. From the first line we obtain $p\left(X, \lambda^{1}\right)=$ $p\left(X, \lambda^{2}\right)=p\left(X, \lambda^{3}\right)=1 / 12$. Combining this with the second, we find $p\left(X, \lambda^{4}\right)=-1 / 12$. Thus, $p$ fails to be non-negative and $\sigma \notin \Sigma$.

Proposition 3.2 gives a necessary and sufficient condition for a finite set $R \subseteq \Delta(\Omega)^{n}$ to be a subset of $R(\sigma)$ for some $\sigma \in \Sigma$. We will now provide a necessary and sufficient condition for such a set $R$ to coincide with $R(\sigma)$ for some $\sigma \in \Sigma$.

Proposition 6.5. Let the non-empty and finite $R \subseteq \Delta(\Omega)^{n}$ be such that, for every $i \in N$, $\left|S_{i}\right| \geq\left|R_{i}\right|$. There exists $\sigma \in \Sigma$ with $R(\sigma)=R$ if and only if there is $p \in \mathbb{R}_{+}^{\Omega \times R}$ such that

(i) $\sum_{\lambda \in R} p(\omega, \lambda)=\lambda^{0}(\omega)$

$\omega \in \Omega$,

(ii) $\sum_{\lambda^{\prime} \in R: \lambda_{i}^{\prime}=\lambda_{i}} p\left(\omega, \lambda^{\prime}\right)=\lambda_{i}(\omega) \sum_{\omega^{\prime} \in \Omega} \sum_{\lambda^{\prime} \in R: \lambda_{i}^{\prime}=\lambda_{i}} p\left(\omega^{\prime}, \lambda^{\prime}\right), \quad \omega \in \Omega, i \in N, \lambda_{i} \in R_{i}$.

If there is $\sigma \in \Sigma$ with $R(\sigma)=R$, then the communication strategy $\pi: \Omega \rightarrow \Delta\left(\Delta(\Omega)^{n}\right)$ defined by

$$
\pi(\lambda \mid \omega)=\frac{p(\omega, \lambda)}{\lambda^{0}(\omega)}, \quad \omega \in \Omega, \lambda \in R(\sigma)
$$

is an LICS such that $R\left(\sigma^{\pi}\right)=R$. 
Proof. Assume that $(i)$ and $(i i)$ hold. Let $\pi: \Omega \rightarrow \Delta\left(\Delta(\Omega)^{n}\right)$ be as defined in (11). We have that

$$
\sum_{\lambda^{\prime} \in R} \pi\left(\lambda^{\prime} \mid \omega\right) \stackrel{(11)}{=} \sum_{\lambda^{\prime} \in R} \frac{p\left(\omega, \lambda^{\prime}\right)}{\lambda^{0}(\omega)} \stackrel{(i)}{=} \frac{\lambda^{0}(\omega)}{\lambda^{0}(\omega)}=1, \quad \omega \in \Omega .
$$

Moreover, for every $\omega \in \Omega, i \in N$, and $\lambda_{i} \in R_{i}$, it holds that

$$
\begin{aligned}
& \frac{\sum_{\lambda^{\prime} \in R: \lambda_{i}^{\prime}=\lambda_{i}} \pi\left(\lambda^{\prime} \mid \omega\right) \lambda^{0}(\omega)}{\sum_{\omega^{\prime} \in \Omega} \sum_{\lambda^{\prime} \in R: \lambda_{i}^{\prime}=\lambda_{i}} \pi\left(\lambda^{\prime} \mid \omega^{\prime}\right) \lambda^{0}\left(\omega^{\prime}\right)} \stackrel{(11)}{=} \frac{\sum_{\lambda^{\prime} \in R: \lambda_{i}^{\prime}=\lambda_{i}} p\left(\omega, \lambda^{\prime}\right)}{\sum_{\omega^{\prime} \in \Omega} \sum_{\lambda^{\prime} \in R: \lambda_{i}^{\prime}=\lambda_{i}} p\left(\omega^{\prime}, \lambda^{\prime}\right)} \\
& \stackrel{(i i)}{=} \frac{\lambda_{i}(\omega) \sum_{\omega^{\prime} \in \Omega} \sum_{\lambda^{\prime} \in R: \lambda_{i}^{\prime}=\lambda_{i}} p\left(\omega^{\prime}, \lambda^{\prime}\right)}{\sum_{\omega^{\prime} \in \Omega} \sum_{\lambda^{\prime} \in R: \lambda_{i}^{\prime}=\lambda_{i}} p\left(\omega^{\prime}, \lambda^{\prime}\right)}=\lambda_{i}(\omega) \text {. }
\end{aligned}
$$

Thus, $\pi$ is an LICS such that $R\left(\sigma^{\pi}\right)=R$.

Finally, for every $i \in N$, let $T_{i}$ be a subset of $S_{i}$ with cardinality equal to $\left|R_{i}\right|$ and take a bijection $\psi_{i}: R_{i} \rightarrow T_{i}$. The communication strategy $\pi^{\prime}: \Omega \rightarrow \Delta(S)$ is defined by

$$
\pi^{\prime}(\psi(\lambda) \mid \omega)=\pi(\lambda \mid \omega), \quad \omega \in \Omega, \lambda \in R .
$$

It holds that $\pi \sim \pi^{\prime}$, so by Proposition 5.7 we have that $\sigma^{\pi^{\prime}}=\sigma^{\pi}$ and $R\left(\sigma^{\pi^{\prime}}\right)=R$.

Now assume that $\sigma \in \Sigma$ is such that $R(\sigma)=R$. Then, by Theorem $6.2, \pi: \Omega \rightarrow$ $\Delta\left(\Delta(\Omega)^{n}\right)$ defined as in (11) is an LICS that induces $\sigma$. Then $(i)$ is satisfied since

$$
\sum_{\lambda^{\prime} \in R} p\left(\omega, \lambda^{\prime}\right) \stackrel{(11)}{=} \sum_{\lambda^{\prime} \in R} \pi\left(\lambda^{\prime} \mid \omega\right) \lambda^{0}(\omega)=\lambda^{0}(\omega) \sum_{\lambda^{\prime} \in S^{\pi}} \pi\left(\lambda^{\prime} \mid \omega\right)=\lambda^{0}(\omega), \quad \omega \in \Omega .
$$

Further, for every $\omega \in \Omega, i \in N$, and $\lambda_{i} \in R_{i}$, it holds that

$$
\begin{aligned}
\sum_{\lambda^{\prime} \in R: \lambda_{i}^{\prime}=\lambda_{i}} p\left(\omega, \lambda^{\prime}\right) & \stackrel{(11)}{=} \sum_{\lambda^{\prime} \in R: \lambda_{i}^{\prime}=\lambda_{i}} \pi\left(\lambda^{\prime} \mid \omega\right) \lambda^{0}(\omega)=\pi_{i}\left(\lambda_{i} \mid \omega\right) \lambda^{0}(\omega) \\
& \stackrel{(1)}{=} \lambda_{i}(\omega) \sum_{\omega^{\prime} \in \Omega} \pi_{i}\left(\lambda_{i} \mid \omega^{\prime}\right) \lambda^{0}\left(\omega^{\prime}\right)=\lambda_{i}(\omega) \sum_{\omega^{\prime} \in \Omega} \sum_{\lambda^{\prime} \in R: \lambda_{i}^{\prime}=\lambda_{i}} \pi\left(\lambda^{\prime} \mid \omega^{\prime}\right) \lambda^{0}\left(\omega^{\prime}\right) \\
& \stackrel{(11)}{=} \lambda_{i}(\omega) \sum_{\omega^{\prime} \in \Omega} \sum_{\lambda^{\prime} \in R: \lambda_{i}^{\prime}=\lambda_{i}} p\left(\omega^{\prime}, \lambda^{\prime}\right) .
\end{aligned}
$$

Hence, $(i i)$ is satisfied.

As $\pi$ is defined by (11), (i) ensures that $\pi(\cdot \mid \omega) \in \Delta(\Omega)^{n}$ for all $\omega \in \Omega$ and $\pi$ is, hence, a communication strategy. Condition $(i i)$ ensures correct belief updating: as before the left-hand side is the probability that $i$ has belief $\lambda_{i}$ and the true state is $\omega$; the right-hand side is the product of the probability that the state is $\omega$ conditional on $i$ 's having belief $\lambda_{i}$ and the probability that $i$ has belief $\lambda_{i}$. 
In our discussion of Proposition 5.7, stating that equivalent communication strategies induce the same distribution, we announced that the converse be not true. We can now easily provide the required counterexample.

Example 6.6. Let $N=\{1,2\}, \Omega=\{X, Y\}, \lambda^{0}(X)=1 / 3$, and $S=\Delta(\Omega)^{n}$. Consider the distribution $\sigma$ defined by

$$
\begin{aligned}
R\left(\sigma^{\pi}\right) & =\left\{\lambda^{1}, \lambda^{2}, \lambda^{3}, \lambda^{4}\right\} \\
& =\left\{\left(\left(\frac{1}{2}, \frac{1}{2}\right),\left(\frac{1}{2}, \frac{1}{2}\right)\right)\left(\left(\frac{1}{2}, \frac{1}{2}\right),\left(\frac{1}{4}, \frac{3}{4}\right)\right),\left(\left(\frac{1}{4}, \frac{3}{4}\right),\left(\frac{1}{2}, \frac{1}{2}\right)\right),\left(\left(\frac{1}{4}, \frac{3}{4}\right),\left(\frac{1}{4}, \frac{3}{4}\right)\right)\right\},
\end{aligned}
$$

$\sigma\left(\lambda^{1}\right)=\sigma\left(\lambda^{2}\right)=\sigma\left(\lambda^{3}\right)=1 / 6$ and $\sigma\left(\lambda^{4}\right)=1 / 2$. One can easily verify that $p, p^{\prime} \in \mathbb{R}_{+}^{\Omega \times R(\sigma)}$

\begin{tabular}{|c|c|c|c|c|c|c|c|c|c|}
\hline$p(\omega, \lambda)$ & $\lambda^{1}$ & $\lambda^{2}$ & $\lambda^{3}$ & $\lambda^{4}$ & $p^{\prime}(\omega, \lambda)$ & $\lambda^{1}$ & $\lambda^{2}$ & $\lambda^{3}$ & $\lambda^{4}$ \\
\hline$X$ & $\frac{1}{12}$ & $\frac{1}{12}$ & $\frac{1}{12}$ & $\frac{1}{12}$ & $X$ & $\frac{1}{6}$ & 0 & 0 & 1 \\
\hline$Y$ & $\frac{1}{12}$ & $\frac{1}{12}$ & $\frac{1}{12}$ & $\frac{5}{12}$ & $Y$ & 0 & $\frac{1}{6}$ & $\frac{1}{6}$ & $\frac{\pi}{3}$ \\
\hline
\end{tabular}
defined by

are both solutions to the system of equations in Theorem 6.2 . We define $\pi, \pi^{\prime} \in \Pi^{\ell}$ by applying (8) to $p$ and $p^{\prime}$, respectively, that is,

\begin{tabular}{c|cccc}
$\pi(\lambda \mid \omega)$ & $\lambda^{1}$ & $\lambda^{2}$ & $\lambda^{3}$ & $\lambda^{4}$ \\
\hline$X$ & $\frac{1}{4}$ & $\frac{1}{4}$ & $\frac{1}{4}$ & $\frac{1}{4}$ \\
$Y$ & $\frac{1}{8}$ & $\frac{1}{8}$ & $\frac{1}{8}$ & $\frac{5}{8}$
\end{tabular}

\begin{tabular}{c|cccc}
$\pi^{\prime}(\lambda \mid \omega)$ & $\lambda^{1}$ & $\lambda^{2}$ & $\lambda^{3}$ & $\lambda^{4}$ \\
\hline$X$ & $\frac{1}{2}$ & 0 & 0 & $\frac{1}{2}$ \\
$Y$ & 0 & $\frac{1}{4}$ & $\frac{1}{4}$ & $\frac{1}{2}$
\end{tabular}

Both $\pi$ and $\pi^{\prime}$ induce $\sigma$. Yet, as $\pi \neq \pi^{\prime}$, Proposition 5.8 implies that $\pi$ and $\pi^{\prime}$ are not equivalent.

\section{The Information and Posterior Correspondences}

Our objective in this section is to provide a framework in which we can analyze what receivers know about each other's messages, so that we can later answer the question of how a sender can make sure that receivers know "as little as possible".

Given a communication strategy $\pi \in \Pi$, we refer to an element $(\omega, s) \in \Omega \times S^{\pi}$ such that $\pi(s \mid \omega)>0$ as a history and to an element $(\omega, \lambda) \in \Omega \times R\left(\sigma^{\pi}\right)$ such that there exists $s \in S^{\pi}$ with $\pi(s \mid \omega)>0$ and $\lambda^{s}=\lambda$ as a posterior history. We denote the sets of histories and posterior histories, respectively, by

$$
\begin{aligned}
H^{\pi} & =\left\{(\omega, s) \in \Omega \times S^{\pi} \mid \pi(s \mid \omega)>0\right\}, \\
\Lambda^{\pi} & =\left\{(\omega, \lambda) \in \Omega \times \Delta(\Omega)^{n} \mid \exists s \in S^{\pi} \text { such that } \pi(s \mid \omega)>0 \text { and } \lambda^{s}=\lambda\right\} .
\end{aligned}
$$

Note that if $\pi \in \Pi^{\ell}$, then $H^{\pi}=\Lambda^{\pi}$. 
Example 7.1. Recall $\pi$ and $\pi^{\prime}$ from Example 6.6. The sets of possible histories are:

$$
\begin{aligned}
H^{\pi} & =\left\{\left(X, \lambda^{1}\right),\left(X, \lambda^{2}\right),\left(X, \lambda^{3}\right),\left(X, \lambda^{4}\right),\left(Y, \lambda^{1}\right),\left(Y, \lambda^{2}\right),\left(Y, \lambda^{3}\right),\left(Y, \lambda^{4}\right)\right\} \\
H^{\pi^{\prime}} & =\left\{\left(X, \lambda^{1}\right),\left(X, \lambda^{4}\right),\left(Y, \lambda^{2}\right),\left(Y, \lambda^{3}\right),\left(Y, \lambda^{4}\right)\right\} .
\end{aligned}
$$

As both communication strategies are language independent, we have $\Lambda^{\pi}=H^{\pi}$ and $\Lambda^{\pi^{\prime}}=H^{\pi^{\prime}}$.

We next introduce the standard notion of an information correspondence.

Definition 7.2. Let $\pi \in \Pi$. The information correspondence $P_{i}^{\pi}: H^{\pi} \rightrightarrows H^{\pi}$ of $i \in N$ is defined as

$$
P_{i}^{\pi}(\omega, s)=\left\{\left(\omega^{\prime}, s^{\prime}\right) \in H^{\pi} \mid s_{i}^{\prime}=s_{i}\right\}, \quad(\omega, s) \in H^{\pi} .
$$

That is, $P_{i}^{\pi}(\omega, s)$ is the set of histories receiver $i$ considers possible when the true history is $(\omega, s)$. As we call $P_{i}^{\pi}$ an information correspondence, it seems appropriate to briefly show that this name is deserved, i.e., consistent with the common definition of an information correspondence, see for instance Osborne and Rubinstein (1994).

Lemma 7.3. Let $\pi \in \Pi$ and $i \in N$. The information correspondence $P_{i}^{\pi}$ satisfies the following two conditions:

C1 For all $(\omega, s) \in H^{\pi},(\omega, s) \in P_{i}^{\pi}(\omega, s)$.

C2 If $\left(\omega^{\prime}, s^{\prime}\right) \in P_{i}^{\pi}(\omega, s)$, then $P_{i}^{\pi}\left(\omega^{\prime}, s^{\prime}\right)=P_{i}^{\pi}(\omega, s)$.

Proof. Let $(\omega, s) \in H^{\pi}$. Suppose $(\omega, s) \notin P_{i}^{\pi}(\omega, s)$. Then, $s_{i} \neq s_{i}$, a contradiction. Thus, C1 is satisfied.

Next, let $\left(\omega^{\prime}, s^{\prime}\right) \in P_{i}^{\pi}(\omega, s)$ and $\left(\omega^{\prime \prime}, s^{\prime \prime}\right) \in P_{i}^{\pi}\left(\omega^{\prime}, s^{\prime}\right)$. Then, $s_{i}^{\prime \prime}=s_{i}^{\prime}=s_{i}$, so $\left(\omega^{\prime \prime}, s^{\prime \prime}\right) \in$ $P_{i}^{\pi}(\omega, s)$, and consequently, $P_{i}^{\pi}\left(\omega^{\prime}, s^{\prime}\right) \subseteq P_{i}^{\pi}(\omega, s)$. Since $s_{i}^{\prime}=s_{i}$, it holds that $(\omega, s) \in$ $P_{i}^{\pi}\left(\omega^{\prime}, s^{\prime}\right)$ as well, and the same arguments imply $P_{i}^{\pi}(\omega, s) \subseteq P_{i}^{\pi}\left(\omega^{\prime}, s^{\prime}\right)$. So, C2 is satisfied.

Information correspondences have the property that they partition sets of histories into information sets. In our case we can use $P_{i}^{\pi}$ to define a partition of the set $H^{\pi}$ as

$$
\mathcal{P}_{i}^{\pi}=\left\{P_{i}^{\pi}(\omega, s) \mid(\omega, s) \in H^{\pi}\right\} .
$$

This partition reflects $i$ 's knowledge about the true history: whenever the true history is $(\omega, s), i$ knows that the true history lies in $P_{i}^{\pi}(\omega, s)$. 
Example 7.4. Recall $\pi$ in Example 4.2. The information correspondence partitions the set of histories as follows:

$$
\begin{aligned}
P_{1}^{\pi}(X,(v, x))=P_{1}^{\pi}(Y,(v, y)) & =\{(X,(v, x)),(Y,(v, y))\}, \\
P_{1}^{\pi}(X,(w, w))=P_{1}^{\pi}(Y,(w, w)) & =\{(X,(w, w)),(Y,(w, w))\}, \\
P_{2}^{\pi}(X,(v, x)) & =\{(X,(v, x)\}, \\
P_{2}^{\pi}(Y,(v, y)) & =\{(Y,(v, y))\}, \\
P_{2}^{\pi}(X,(w, w))=P_{2}^{\pi}(Y,(w, w)) & =\{(X,(w, w)),(Y,(w, w))\} .
\end{aligned}
$$

Now consider $\pi^{\prime}$ in Example 4.4. The information correspondence partitions the set of histories as follows:

$$
\begin{aligned}
P_{1}^{\pi^{\prime}}(X,(w, x)) & =P_{1}^{\pi^{\prime}}(Y,(w, y))=P_{1}^{\pi^{\prime}}(X,(w, w))=P_{1}^{\pi^{\prime}}(Y,(w, w)) \\
& =\{(X,(w, x)),(Y,(w, y)),(X,(w, w)),(Y,(w, w))\}, \\
P_{2}^{\pi^{\prime}}(X,(w, x)) & =\{(X,(w, x)\}, \\
P_{2}^{\pi^{\prime}}(Y,(w, y)) & =\{(Y,(w, y))\}, \\
P_{2}^{\pi^{\prime}}(X,(w, w))=P_{2}^{\pi^{\prime}}(Y,(w, w)) & =\{(X,(w, w)),(Y,(w, w))\} .
\end{aligned}
$$

It is easy to verify that both $\mathrm{C} 1$ and $\mathrm{C} 2$ are satisfied. In particular, the information partitions of $\mathcal{P}_{i}^{\pi}$ and, respectively, $\mathcal{P}_{i}^{\pi^{\prime}}$ are given by

$$
\begin{aligned}
\mathcal{P}_{1}^{\pi} & =\{\{(X,(v, x)),(Y,(v, y))\},\{(X,(w, w)),(Y,(w, w))\}\}, \\
\mathcal{P}_{2}^{\pi} & =\{\{(X,(v, x))\},\{(Y,(v, y))\},\{(X,(w, w)),(Y,(w, w))\}\}, \\
\mathcal{P}_{1}^{\pi^{\prime}} & =\{\{(X,(w, x)),(Y,(w, y)),(X,(w, w)),(Y,(w, w))\}\}, \\
\mathcal{P}_{2}^{\pi^{\prime}} & =\{\{(X,(w, x))\},\{(Y,(w, y))\},\{(X,(w, w)),(Y,(w, w))\}\} .
\end{aligned}
$$

Even though $\pi$ and $\pi^{\prime}$ in Example 7.4 induce the same distribution, it is not possible to compare their information partitions since they employ different messages and thus have distinct sets of histories. Still, we can compare such communication strategies via the sets of possible posterior histories of receivers.

Definition 7.5. Let $\pi \in \Pi$. The posterior correspondence $Q_{i}^{\pi}: H^{\pi} \rightrightarrows \Lambda^{\pi}$ of $i \in N$ is defined as

$$
Q_{i}^{\pi}(\omega, s)=\left\{\left(\omega^{\prime}, \lambda^{s^{\prime}}\right) \in \Lambda^{\pi} \mid\left(\omega^{\prime}, s^{\prime}\right) \in P_{i}^{\pi}(\omega, s)\right\}, \quad(\omega, s) \in H^{\pi}
$$


The set $Q_{i}^{\pi}(\omega, s)$ contains all posterior histories $i$ deems possible if the true history is $(\omega, s)$.

Example 7.6. Recall the information correspondences in Example 7.4. The posterior correspondences related to $\pi$ are as follows.

$$
\begin{aligned}
Q_{1}^{\pi}(X,(t, x)) & =\left\{\left(X,\left(\frac{1}{2}, 1\right)\right),\left(Y,\left(\frac{1}{2}, 0\right)\right)\right\}, \\
Q_{1}^{\pi}(X,(w, w)) & =\left\{\left(X,\left(\frac{1}{2}, \frac{1}{2}\right)\right),\left(Y,\left(\frac{1}{2}, \frac{1}{2}\right)\right)\right\}, \\
Q_{2}^{\pi}(X,(t, x)) & =\left\{\left(X,\left(\frac{1}{2}, 1\right)\right)\right\}, \\
Q_{2}^{\pi}(Y,(t, y)) & =\left\{\left(Y,\left(\frac{1}{2}, 0\right)\right)\right\}, \\
Q_{2}^{\pi}(X,(w, w)) & =\left\{\left(X,\left(\frac{1}{2}, \frac{1}{2}\right)\right),\left(Y,\left(\frac{1}{2}, \frac{1}{2}\right)\right)\right\} .
\end{aligned}
$$

The posterior correspondences related to $\pi^{\prime}$ are as follows.

$$
\begin{aligned}
Q_{1}^{\pi^{\prime}}(X,(w, x)) & =\left\{\left(X,\left(\frac{1}{2}, 1\right)\right),\left(Y,\left(\frac{1}{2}, 0\right)\right),\left(X,\left(\frac{1}{2}, \frac{1}{2}\right)\right),\left(Y,\left(\frac{1}{2}, \frac{1}{2}\right)\right)\right\}, \\
Q_{2}^{\pi^{\prime}}(X,(w, x)) & =\left\{\left(X,\left(\frac{1}{2}, 1\right)\right)\right\}, \\
Q_{2}^{\pi^{\prime}}(Y,(w, y)) & =\left\{\left(Y,\left(\frac{1}{2}, 0\right)\right)\right\}, \\
Q_{2}^{\pi^{\prime}}(X,(w, w)) & =\left\{\left(X,\left(\frac{1}{2}, \frac{1}{2}\right)\right),\left(Y,\left(\frac{1}{2}, \frac{1}{2}\right)\right)\right\} .
\end{aligned}
$$

One can easily see that there is a bijection between the set of possible histories and the set of possible posterior histories for both $\pi$ and $\pi^{\prime}$.

For $\pi \in \Pi$ and $i \in N$, define $\mathcal{Q}_{i}^{\pi}=\left\{Q_{i}^{\pi}(\omega, s) \mid(\omega, s) \in H^{\pi}\right\}$. Note that in Example 7.6 both $\mathcal{Q}_{i}^{\pi}$ and $\mathcal{Q}_{i}^{\pi^{\prime}}$ are partitions for any $i \in N$. However, this is not always true.

Example 7.7. Let $N=\{1,2\}, \Omega=\{X, Y\}$, and $\lambda^{0}(X)=1 / 3$. Let communication strategy $\pi \in \Pi$ be given as follows:

\begin{tabular}{c|cccccccc}
$\pi$ & $(x, x)$ & $(x, y)$ & $(y, x)$ & $(y, y)$ & $(a, a)$ & $(a, b)$ & $(b, a)$ & $(b, b)$ \\
\hline$X$ & $\frac{1}{6}$ & 0 & 0 & $\frac{1}{6}$ & $\frac{1}{6}$ & $\frac{1}{6}$ & $\frac{1}{6}$ & $\frac{1}{6}$ \\
$Y$ & 0 & $\frac{1}{12}$ & $\frac{1}{12}$ & $\frac{1}{6}$ & $\frac{1}{12}$ & $\frac{1}{12}$ & $\frac{1}{12}$ & $\frac{5}{12}$
\end{tabular}

For the posterior correspondence we find

$$
\begin{aligned}
& Q_{1}^{\pi}(X,(x, x))=\left\{\left(X,\left(\frac{1}{2}, \frac{1}{2}\right)\right),\left(Y,\left(\frac{1}{2}, \frac{1}{4}\right)\right)\right\}, \\
& Q_{1}^{\pi}(X,(a, a))=\left\{\left(X,\left(\frac{1}{2}, \frac{1}{2}\right)\right),\left(X,\left(\frac{1}{2}, \frac{1}{4}\right)\right),\left(Y,\left(\frac{1}{2}, \frac{1}{2}\right)\right),\left(Y,\left(\frac{1}{2}, \frac{1}{4}\right)\right)\right\} .
\end{aligned}
$$

Since $Q_{1}^{\pi}(X,(x, x)) \neq Q_{1}^{\pi}(X,(a, a))$ and $(X,(1 / 2,1 / 2)) \in Q_{1}^{\pi}(X,(x, x)) \cap Q_{1}^{\pi}(X,(a, a))$, $\mathcal{Q}_{1}^{\pi}$ is not a partition. 
The reason why $Q_{1}^{\pi}$ in Example 7.7 is not a partition is that signals $(x, x)$ and $(a, a)$ lead to the same posterior belief vector, yet $(x, x)$ realizes only in state $X$ whereas $(a, a)$ realizes in both states. This situation, of course, can happen only as long as the communication strategy is not minimal. Put differently, $\pi \in \Pi^{\mathrm{m}}$ is sufficient for $\mathcal{Q}_{i}^{\pi}$ to be a partition for all $i \in N$.

For $\pi \in \Pi$, we define the function $\phi: H^{\pi} \rightarrow \Lambda^{\pi}$ by

$$
\phi(\omega, s)=\left(\omega, \lambda^{s}\right), \quad(\omega, s) \in H^{\pi} .
$$

Proposition 7.8. Let $\pi \in \Pi^{\mathrm{m}}$. Then $\phi$ is a bijection and, for every $(\omega, s),\left(\omega^{\prime}, s^{\prime}\right) \in H^{\pi}$ and every $i \in N$, it holds that $(\omega, s) \in P_{i}^{\pi}\left(\omega^{\prime}, s^{\prime}\right)$ if and only if $\phi(\omega, s) \in Q_{i}^{\pi}\left(\omega^{\prime}, s^{\prime}\right)$. In particular, $\mathcal{Q}_{i}^{\pi}$ is a partition.

Proof. First note that since $\pi \in \Pi^{\mathrm{m}}$, for any $(\omega, s),\left(\omega^{\prime}, s^{\prime}\right) \in H^{\pi}$ with $s \neq s^{\prime}$, it holds that $\left(\omega, \lambda^{s}\right) \neq\left(\omega^{\prime}, \lambda^{s^{\prime}}\right)$. That is, no two distinct histories are mapped to the same posterior history. Thus, $\phi$ is a bijection.

Let $(\omega, s),\left(\omega^{\prime}, s^{\prime}\right) \in H^{\pi}$ and $i \in N$. If $(\omega, s) \in P_{i}^{\pi}\left(\omega^{\prime}, s^{\prime}\right)$, then $\phi(\omega, s)=\left(\omega, \lambda^{s}\right) \in$ $Q_{i}^{\pi}\left(\omega^{\prime}, s^{\prime}\right)$ by the definition of $Q_{i}^{\pi}\left(\omega^{\prime}, s^{\prime}\right)$. If $\left(\omega, \lambda^{s}\right)=\phi(\omega, s) \in Q_{i}^{\pi}\left(\omega^{\prime}, s^{\prime}\right)$, then $(\omega, s) \in$ $P_{i}^{\pi}\left(\omega^{\prime}, s^{\prime}\right)$. Therefore, $(\omega, s) \in P_{i}^{\pi}\left(\omega^{\prime}, s^{\prime}\right)$ if and only if $\phi(\omega, s) \in Q_{i}^{\pi}\left(\omega^{\prime}, s^{\prime}\right)$.

Suppose $Q_{i}^{\pi}(\omega, s) \cap Q_{i}^{\pi}\left(\omega^{\prime}, s^{\prime}\right) \neq \emptyset$. It follows that $P_{i}^{\pi}(\omega, s) \cap P_{i}^{\pi}\left(\omega^{\prime}, s^{\prime}\right) \neq \emptyset$, so $P_{i}^{\pi}(\omega, s)=P_{i}^{\pi}\left(\omega^{\prime}, s^{\prime}\right)$. Therefore, $Q_{i}^{\pi}(\omega, s)=\phi\left(P_{i}^{\pi}(\omega, s)\right)=\phi\left(P_{i}^{\pi}\left(\omega^{\prime}, s^{\prime}\right)\right)=Q_{i}^{\pi}\left(\omega^{\prime}, s^{\prime}\right)$, so $\mathcal{Q}_{i}^{\pi}$ is a partition.

The converse of Proposition 7.8 is not true. That is, even if the map $\phi$ is a bijection with the required properties, it is still possible that $\pi$ is not minimal.

Example 7.9. Let $N=\{1,2\}, \Omega=\{X, Y\}$, and $\lambda^{0}(X)=1 / 3$. The communication strategy $\pi \in \Pi$ is defined by

\begin{tabular}{c|ccccccc}
$\pi$ & $(a, a)$ & $(b, b)$ & $(a, c)$ & $(c, a)$ & $(b, d)$ & $(d, b)$ & $(e, e)$ \\
\hline$X$ & $\frac{1}{6}$ & 0 & 0 & 0 & $\frac{1}{4}$ & $\frac{1}{6}$ & $\frac{5}{12}$ \\
$Y$ & 0 & $\frac{1}{4}$ & $\frac{1}{6}$ & $\frac{1}{4}$ & 0 & 0 & $\frac{1}{3}$
\end{tabular}

Then, for receiver 1 we have $\lambda_{1}^{(a, a)}(X)=\lambda_{1}^{(b, b)}(X)=1 / 3, \lambda_{1}^{(c, a)}(X)=0, \lambda_{1}^{(d, b)}(X)=1$, and $\lambda_{1}^{(e, e)}(X)=5 / 13$. For receiver 2 we have $\lambda_{2}^{(a, a)}(X)=\lambda_{2}^{(b, b)}(X)=1 / 4, \lambda_{2}^{(a, c)}(X)=0$, $\lambda_{2}^{(b, d)}(X)=1$, and $\lambda_{2}^{(e, e)}(X)=5 / 13$. Note that signals $(a, a)$ and $(b, b)$ lead to the same posterior belief vector, $(1 / 3,1 / 4)$. Thus, $\pi$ is not minimal. For the support of the induced distribution $\sigma$ we find

$$
R(\sigma)=\left\{\left(\frac{1}{3}, \frac{1}{4}\right),\left(\frac{1}{3}, 0\right),\left(0, \frac{1}{4}\right),\left(\frac{1}{3}, 1\right),\left(1, \frac{1}{4}\right),\left(\frac{5}{13}, \frac{5}{13}\right)\right\} .
$$




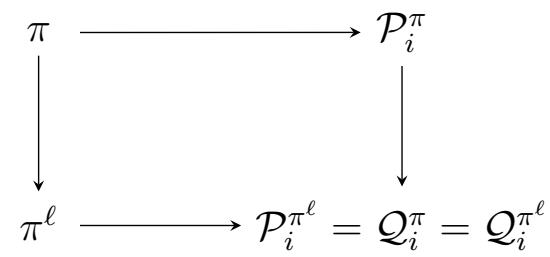

Figure 1: Commuting Diagram for $\pi \in \Pi^{\mathrm{d}}$, see Corollary 7.10.

The sets $\mathcal{P}_{1}^{\pi}$ and $\mathcal{Q}_{1}^{\pi}$ defined by the information and posterior correspondence of receiver 1 are as follows:

$$
\begin{aligned}
& \mathcal{P}_{1}^{\pi}=\{(X,(a, a)),(Y,(a, c))\},\{(Y,(c, a))\},\{(X,(b, d)),(Y,(b, b))\},\{(X,(d, b))\}, \\
&\{(X,(e, e)),(Y,(e, e))\}\}, \\
& \mathcal{Q}_{1}^{\pi}=\left\{\left\{\left(X,\left(\frac{1}{3}, \frac{1}{4}\right)\right),\left(Y,\left(\frac{1}{3}, 0\right)\right)\right\},\left\{\left(Y,\left(0, \frac{1}{4}\right)\right)\right\},\left\{\left(X,\left(\frac{1}{3}, 1\right)\right),\left(Y,\left(\frac{1}{3}, \frac{1}{4}\right)\right)\right\},\left\{\left(X,\left(1, \frac{1}{4}\right)\right)\right\},\right. \\
&\left.\left\{\left(X,\left(\frac{5}{13}, \frac{5}{13}\right)\right),\left(Y,\left(\frac{5}{13}, \frac{5}{13}\right)\right)\right\}\right\} .
\end{aligned}
$$

Similar calculations can be made for receiver 2 . It is easily checked that not only are $\mathcal{Q}_{1}^{\pi}$ and $\mathcal{Q}_{2}^{\pi}$ partitions, but $\phi$ is a bijection as well. The reason $\mathcal{Q}_{1}^{\pi}$ and $\mathcal{Q}_{2}^{\pi}$ are partitions, even though $\pi \notin \Pi^{\mathrm{m}}$, is that the signals which lead to the same posterior, $(a, a)$ and $(b, b)$, never realize in the same state.

Observe that if $\pi \in \Pi^{\ell}$, then $\phi$ is the identity. Hence, the proposition implies that the partitions $\mathcal{P}_{i}^{\pi}$ and $\mathcal{Q}_{i}^{\pi}$ are identical. For all $\pi \in \Pi^{\mathrm{d}}$, let $\pi^{\ell} \in \Pi^{\ell}$ be defined as in (7), i.e., $\pi^{\ell}$ denotes the LICS obtained by replacing the messages of $\pi$ by the posteriors they lead to. Then the posterior history partition of $\pi$ is equal to the history partition of $\pi^{\ell}$. Thus, we have the following corollary which is depicted in the diagram in Figure 1.

Corollary 7.10. Let $\pi \in \Pi^{\mathrm{d}}$ and $\pi^{\ell} \in \Pi^{\ell}$ be defined as in (7). Then, for all $i \in N$, $\mathcal{Q}_{i}^{\pi}=\mathcal{Q}_{i}^{\pi^{\ell}}=\mathcal{P}_{i}^{\pi^{\ell}}$.

\section{Informativeness of Communication Strategies}

Example 7.6 derives the posterior correspondences of the receivers under $\pi$ and $\pi^{\prime}$ from Examples 4.2 and 4.4. Observe that receiver 1 has more precise information about receiver 2's knowledge of the true state under $\pi$ : while he only observes $w$ under $\pi^{\prime}$ and, thus, never learns what message receiver 2 has observed, under $\pi$ upon observing $v$ he knows that receiver 2 knows the true state. In this sense $\pi$ is "more informative": a notion that depends on the posterior correspondence and which we will make more formal soon. Beforehand, we make the brief observation that the posterior correspondence 
itself is invariant under equivalence or, put differently, that the posterior correspondence is language independent.

Lemma 8.1. Let $\pi, \pi^{\prime} \in \Pi$ with $\pi \sim \pi^{\prime}$. Then, for every $i \in N, \mathcal{Q}_{i}^{\pi}=\mathcal{Q}_{i}^{\pi^{\prime}}$.

Proof. Since $\pi \sim \pi^{\prime}$, for every $i \in N$ there is a bijection $\psi_{i}: S_{i}^{\pi} \rightarrow S_{i}^{\pi^{\prime}}$ such that, for every $\omega \in \Omega$, for every $s \in S^{\pi}, \pi^{\prime}(\psi(s) \mid \omega)=\pi(s \mid \omega)$.

Let $(\omega, s) \in H^{\pi}$ and $i \in N$.

We have that $\left(\omega^{\prime}, s^{\prime}\right) \in P_{i}^{\pi}(\omega, s)$ if and only if $\left(\omega^{\prime}, s^{\prime}\right) \in H^{\pi}$ and $s_{i}^{\prime}=s_{i}$ if and only if $\left(\omega^{\prime}, \psi\left(s^{\prime}\right)\right) \in H^{\pi^{\prime}}$ and $\psi_{i}\left(s_{i}^{\prime}\right)=\psi_{i}\left(s_{i}\right)$ if and only if $\left(\omega^{\prime}, \psi\left(s^{\prime}\right)\right) \in P_{i}^{\pi^{\prime}}(\omega, \psi(s))$.

Let $\left(\omega^{\prime}, \lambda^{\prime}\right) \in Q_{i}^{\pi^{\prime}}(\omega, \psi(s))$. Then, by the definition of $Q_{i}^{\pi^{\prime}}$, there is $\left(\omega^{\prime}, \psi\left(s^{\prime}\right)\right) \in$ $P_{i}^{\pi^{\prime}}(\omega, \psi(s))$ with $\lambda^{\prime^{\psi}\left(s^{\prime}\right)}=\lambda^{\prime}$. As shown in the previous paragraph, this implies $\left(\omega^{\prime}, s^{\prime}\right) \in$ $P_{i}^{\pi}(\omega, s)$ and $\lambda^{s^{\prime}}=\lambda^{\psi^{\psi}\left(s^{\prime}\right)}=\lambda^{\prime}$. It follows that $\left(\omega^{\prime}, \lambda^{\prime}\right) \in Q^{\pi}(\omega, s)$ and therefore $Q_{i}^{\pi^{\prime}}(\omega, \psi(s)) \subseteq Q_{i}^{\pi}(\omega, s)$.

Since $\sim$ is reflexive, we also have that $Q_{i}^{\pi}(\omega, s) \subseteq Q_{i}^{\pi^{\prime}}(\omega, \psi(s))$.

We argued in Example 7.6 that the communication strategy $\pi$ is "more informative" for receiver 1 than communication strategy $\pi^{\prime}$. We now give a precise definition of being more informative.

Definition 8.2. Let $\sigma \in \Sigma$ and $\pi, \pi^{\prime} \in \Pi(\sigma)$. The communication strategy $\pi^{\prime}$ is at least as informative as $\pi$ if for all $i \in N$ it holds that

(i) for all $Q^{\prime} \in \mathcal{Q}_{i}^{\pi^{\prime}}$ there exists $Q \in \mathcal{Q}_{i}^{\pi}$ such that $Q^{\prime} \subseteq Q$,

(ii) for all $Q \in \mathcal{Q}_{i}^{\pi}, Q^{\prime} \in \mathcal{Q}_{i}^{\pi^{\prime}}$ with $Q \cap Q^{\prime} \neq \emptyset$ it holds that $Q^{\prime} \subseteq Q$.

Moreover, $\pi$ and $\pi^{\prime}$ are equally informative if $\pi$ is at least as informative as $\pi^{\prime}$ and vice versa; $\pi^{\prime}$ is more informative than $\pi$ if $\pi^{\prime}$ is at least as informative as $\pi$ and not equally informative.

It is easily verified that the notion of being at least as informative is transitive. Our second observation serves as a sanity check: two communication strategies should be equally informative if and only if they induce the same posterior history. And this is true.

Lemma 8.3. Let $\sigma \in \Sigma$ and $\pi, \pi^{\prime} \in \Pi(\sigma)$. Then $\pi$ and $\pi^{\prime}$ are equally informative if and only if $\mathcal{Q}_{i}^{\pi}=\mathcal{Q}_{i}^{\pi^{\prime}}$.

Proof. Clearly, if $\mathcal{Q}_{i}^{\pi}=\mathcal{Q}_{i}^{\pi^{\prime}}$ then $\pi$ and $\pi^{\prime}$ are equally informative. For the other direction, assume that $\pi$ and $\pi^{\prime}$ are equally informative. As $\pi^{\prime}$ is as informative as $\pi$, for all $Q^{\prime} \in \mathcal{Q}_{i}^{\pi^{\prime}}$ there is $Q \in \mathcal{Q}_{i}^{\pi}$ such that $Q^{\prime} \subseteq Q$. As $Q^{\prime} \cap Q \neq \emptyset$ and as $\pi$ is as informative as $\pi^{\prime}$, it must hold that $Q \subseteq Q^{\prime}$, i.e., $Q^{\prime}=Q$. Thus, $\mathcal{Q}_{i}^{\pi^{\prime}} \subseteq \mathcal{Q}_{i}^{\pi}$. Using the same arguments one finds $\mathcal{Q}_{i}^{\pi} \subseteq \mathcal{Q}_{i}^{\pi^{\prime}}$. 
Two further observations on informativeness that are worth mentioning here. First, if $\pi^{\prime}$ is at least as informative as $\pi$, then $\Lambda^{\pi^{\prime}} \subseteq \Lambda^{\pi}$. Second, and an immediate consequence of Lemmas 8.1 and 8.3, equivalent communication strategies are equally informative. This is in line with our interpretation of equivalent communication strategies as using different languages: if the same messages were conveyed in different languages, one would not expect them to become more or less informative.

Example 8.4. Recall the communication strategies $\pi$ and $\pi^{\prime}$ from Examples 4.2 and 4.4. The posterior history correspondences of $\pi$ and $\pi^{\prime}$ were derived in Example 7.6. Note that $\Lambda^{\pi}=\Lambda^{\pi^{\prime}}$ and that $\pi, \pi^{\prime} \in \Pi^{\mathrm{m}}$. Thus, Proposition 7.8 implies that, for every $i \in N$, $\mathcal{Q}_{i}^{\pi}$ and $\mathcal{Q}_{i}^{\pi^{\prime}}$ are partitions of the same set. More precisely, they are given as

$$
\begin{aligned}
& \mathcal{Q}_{1}^{\pi}=\left\{\left\{\left(X,\left(\frac{1}{2}, 1\right)\right),\left(Y,\left(\frac{1}{2}, 0\right)\right)\right\},\left\{\left(X,\left(\frac{1}{2}, \frac{1}{2}\right)\right),\left(Y,\left(\frac{1}{2}, \frac{1}{2}\right)\right)\right\}\right\}, \\
& \mathcal{Q}_{2}^{\pi}=\left\{\left\{\left(X,\left(\frac{1}{2}, 1\right)\right)\right\},\left\{\left(Y,\left(\frac{1}{2}, 0\right)\right)\right\},\left\{\left(X,\left(\frac{1}{2}, \frac{1}{2}\right)\right),\left(Y,\left(\frac{1}{2}, \frac{1}{2}\right)\right)\right\}\right\}, \\
& \mathcal{Q}_{1}^{\pi^{\prime}}=\left\{\left\{\left(X,\left(\frac{1}{2}, 1\right)\right),\left(Y,\left(\frac{1}{2}, 0\right)\right),\left(X,\left(\frac{1}{2}, \frac{1}{2}\right)\right),\left(Y,\left(\frac{1}{2}, \frac{1}{2}\right)\right)\right\}\right\}, \\
& \mathcal{Q}_{2}^{\pi^{\prime}}=\left\{\left\{\left(X,\left(\frac{1}{2}, 1\right)\right)\right\},\left\{\left(Y,\left(\frac{1}{2}, 0\right)\right)\right\},\left\{\left(X,\left(\frac{1}{2}, \frac{1}{2}\right)\right),\left(Y,\left(\frac{1}{2}, \frac{1}{2}\right)\right)\right\}\right\} .
\end{aligned}
$$

It holds that $\mathcal{Q}_{1}^{\pi}$ is a finer partition than $\mathcal{Q}_{1}^{\pi^{\prime}}$ and that $\mathcal{Q}_{2}^{\pi}=\mathcal{Q}_{2}^{\pi^{\prime}}$. Thus, $\pi$ is more informative than $\pi^{\prime}$.

Note that we do not require $\mathcal{Q}_{i}^{\pi}$ and $\mathcal{Q}_{i}^{\pi^{\prime}}$ to be partitions in order to compare $\pi$ and $\pi^{\prime}$. Nevertheless, if they are partitions, then $\pi^{\prime}$ is more informative than $\pi$ if the restriction of $\mathcal{Q}_{i}^{\pi}$ to $\Lambda^{\pi^{\prime}}$ is coarser than $\mathcal{Q}_{i}^{\pi^{\prime}}$.

Proposition 8.5. Let $\sigma \in \Sigma, \pi, \pi^{\prime} \in \Pi(\sigma)$, and $\Lambda^{\pi^{\prime}} \subseteq \Lambda^{\pi}$. If $\pi \in \Pi^{\mathrm{d}}$, then $\pi^{\prime}$ is at least as informative as $\pi$.

Proof. By Corollary 7.10 and Lemma 8.1 we can assume without loss of generality that $\pi \in \Pi^{\ell}$, so that $\mathcal{Q}_{i}^{\pi}=\mathcal{P}_{i}^{\pi}$ for all $i \in N$.

Let $i \in N$. Assume $Q \in \mathcal{Q}_{i}^{\pi}$ and $Q^{\prime} \in \mathcal{Q}_{i}^{\pi^{\prime}}$ are such that $Q \cap Q^{\prime} \neq \emptyset$. We first show Condition (ii) of Definition 8.2, i.e., $Q^{\prime} \subseteq Q$. Let $\left(\omega^{*}, \lambda^{*}\right) \in Q \cap Q^{\prime}$. There is $(\omega, \lambda) \in H^{\pi}$ such that $Q=Q_{i}^{\pi}(\omega, \lambda)=P_{i}^{\pi}(\omega, \lambda)$. Thus, by Lemma 7.3, we have that $Q=P_{i}^{\pi}\left(\omega^{*}, \lambda^{*}\right)$. Consider $(\bar{\omega}, \bar{\lambda}) \in Q^{\prime}$. There is $\left(\omega^{\prime}, s^{\prime}\right) \in H^{\pi^{\prime}}$ such that $Q^{\prime}=Q_{i}^{\pi^{\prime}}\left(\omega^{\prime}, s^{\prime}\right)$ and there is $\left(\omega^{\prime \prime}, s^{\prime \prime}\right) \in P_{i}^{\pi^{\prime}}\left(\omega^{\prime}, s^{\prime}\right)$ with $\lambda^{s^{\prime \prime}}=\bar{\lambda}$. In particular, since $s_{i}^{\prime \prime}=s_{i}^{\prime}$, we have $\bar{\lambda}_{i}=\lambda_{i}^{s^{s^{\prime \prime}}}=\lambda_{i}^{s^{\prime \prime}}=\lambda_{i}^{*}$. Since $\Lambda^{\pi^{\prime}} \subseteq \Lambda^{\pi}$, we have $(\bar{\omega}, \bar{\lambda}) \in \Lambda^{\pi}$, and since $\bar{\lambda}_{i}=\lambda_{i}^{*}$, we have $(\bar{\omega}, \bar{\lambda}) \in P_{i}^{\pi}\left(\omega^{*}, \lambda^{*}\right)=Q$. We have shown that $Q^{\prime} \subseteq Q$.

In order to prove Condition (i) of Definition 8.2 it is now sufficient to show that for each $Q^{\prime} \in \mathcal{Q}_{i}^{\pi^{\prime}}$ there is $Q \in \mathcal{Q}_{i}^{\pi}$ with $Q \cap Q^{\prime} \neq \emptyset$. Let $\left(\omega^{\prime}, s^{\prime}\right) \in H^{\pi^{\prime}}$ be such that $Q^{\prime}=Q_{i}^{\pi^{\prime}}\left(\omega^{\prime}, s^{\prime}\right)$. It holds that $\left(\omega^{\prime}, \lambda^{s^{\prime}}\right) \in Q^{\prime} \subseteq \Lambda^{\pi^{\prime}} \subseteq \Lambda^{\pi}$. Thus, there is $Q \in \mathcal{Q}_{i}^{\pi}$ with $\left(\omega^{\prime}, \lambda^{s^{\prime}}\right) \in Q$. 
Proposition 8.5 reveals that among those communication strategies that induce the same distribution over posterior belief vectors, those that are direct and have the largest number of posterior histories are the least informative. We can interpret the condition $\Lambda^{\pi^{\prime}} \subseteq \Lambda^{\pi}$ as $\pi^{\prime}$ providing additional information about what posterior histories are impossible. It is worth mentioning that this condition together with the directness of $\pi$ implies that $\mathcal{Q}_{i}^{\pi^{\prime}}$ contains at least the same number of elements as $\mathcal{Q}_{i}^{\pi}$ and that these elements are smaller in the sense of set inclusion.

Consider $\pi, \pi^{\prime} \in \Pi^{\mathrm{d}}$ that satisfy the conditions of Proposition 8.5. In this case $\Lambda^{\pi^{\prime}} \subsetneq \Lambda^{\pi}$ would prevent $\pi$ from being at least as informative as $\pi^{\prime}$. Thus the following corollary is immediate.

Corollary 8.6. Let $\sigma \in \Sigma$ and $\pi, \pi^{\prime} \in \Pi^{\mathrm{d}}(\sigma)$. If $\Lambda^{\pi^{\prime}}=\Lambda^{\pi}$, then $\pi$ and $\pi^{\prime}$ are equally informative. If $\Lambda^{\pi^{\prime}} \subsetneq \Lambda^{\pi}$, then $\pi^{\prime}$ is more informative than $\pi$.

In Corollary 5.10 a communication strategy is transformed into an LICS that induces the same distribution over posterior vectors. Although they are not equivalent if $\pi$ is not direct, they have the same set of posterior histories as the next lemma shows.

Lemma 8.7. Let $\Delta(\Omega)^{n} \subseteq S$ and $\pi \in \Pi$. For $\pi^{\ell}$ as defined in (7) it holds that $\Lambda^{\pi^{\ell}}=\Lambda^{\pi}$.

Proof. Observe that $(\omega, \lambda) \in \Lambda^{\pi}$ if and only if there is $s \in S^{\pi}$ such that $\lambda=\lambda^{s}$ and $\pi(s \mid \omega)>0$. This, however, is equivalent to $\pi^{\ell}(\lambda \mid \omega)=\sum_{s \in S^{\pi}: \lambda^{s}=\lambda} \pi(s \mid \omega)>0$, which holds if and only if $(\omega, \lambda) \in H^{\pi^{\ell}}=\Lambda^{\pi^{\ell}}$.

Proposition 8.5 and Lemma 8.7 immediately imply the following result.

Corollary 8.8. Let $\Delta(\Omega)^{n} \subseteq S, \pi \in \Pi$, and $\pi^{\ell} \in \Pi^{\ell}$ as defined in (7). Then $\pi$ is at least as informative as $\pi^{\ell}$.

Corollary 8.8 suggests that using language independent communication strategies reveals as little information as possible. The following example demonstrates that this is, in general, not true.

Example 8.9. Recall $\pi$ and $\pi^{\prime}$ from Example 6.6. Both communication strategies are language independent and, hence, direct. However, as shown in Example 7.1, $\Lambda^{\pi^{\prime}}=H^{\pi^{\prime}} \subsetneq$ $H^{\pi}=\Lambda^{\pi}$. Thus, by Proposition 8.5, $\pi^{\prime}$ is more informative than $\pi$. Observe that it is not relevant that $\pi$ is an LICS: when translating each message sent under $\pi$ in two different languages and sending both with equal probability, we obtain a communication strategy that is not even minimal, but equally informative as $\pi$.

Our final result identifies those communication strategies that are least informative. Let $\sigma \in \Sigma$ and recall that the set $P(\sigma)$ is convex. The relative interior of $P(\sigma)$ is defined as

$$
\operatorname{relint}(P(\sigma))=\left\{p \in P(\sigma) \mid \forall p^{\prime} \in P(\sigma), \exists \alpha>1, \alpha p+(1-\alpha) p^{\prime} \in P(\sigma)\right\} \text {. }
$$


Proposition 8.10. Let $\Delta(\Omega)^{n} \subseteq S, \sigma \in \Sigma$, and $\pi \in \Pi(\sigma)$. For every $p \in P(\sigma)$, define the communication strategy $\pi(p) \in \Pi^{\ell}$ by

$$
\pi(\lambda \mid \omega)=\frac{p(\omega, \lambda)}{\lambda^{0}(\omega)}, \quad \omega \in \Omega, \lambda \in R(\sigma) .
$$

If $p \in \operatorname{relint}(\mathrm{P}(\sigma))$, then $\pi$ is at least as informative as $\pi(p)$.

Proof. For every $p \in P(\sigma)$, it holds that $\Lambda^{\pi(p)}=\{(\omega, \lambda) \in \Omega \times R(\sigma) \mid p(\omega, \lambda)>0\}$. If $p \in$ relint $(\mathrm{P}(\sigma))$, then, for every $p^{\prime} \in P(\sigma), \Lambda^{\pi\left(p^{\prime}\right)} \subseteq \Lambda^{\pi(p)}$, so by Corollary 8.6 it holds that $\pi\left(p^{\prime}\right)$ is at least as informative as $\pi(p)$.

Let $\pi^{\ell} \in \Pi^{\ell}$ be as defined in (7) and define $p^{\prime} \in P(\sigma)$ by

$$
p^{\prime}(\omega, \lambda)=\lambda^{0}(\omega) \pi^{\ell}(\lambda \mid \omega), \quad \omega \in \Omega, \lambda \in R(\sigma) .
$$

It follows from Corollary 8.8 that $\pi$ is at least as informative as $\pi^{\ell}=\pi\left(p^{\prime}\right)$, so at least as informative as $\pi(p)$ for every $p \in \operatorname{relint}(P(\sigma))$.

\section{Conclusion}

This paper considers a Bayesian persuasion framework with multiple receivers and investigates $(i)$ the inducible distributions of posterior belief vectors and (ii) informativeness of communication strategies. The sender can restrict attention to particular classes of communication strategies without loss of generality. In particular, any distribution over posterior belief vectors can be induced by a language independent communication strategy. Moreover, any direct communication strategy can be transformed into an equivalent LICS.

Extending Kamenica and Gentzkow (2011) by assuming multiple receivers and private communication imposes further constraints on inducible distributions over posterior belief vectors, so that Bayes plausibility is no longer a sufficient condition. We formulate the additional conditions in the form of a linear system of equations that needs to have a non-negative solution. These conditions, together with Bayes plausibility, are necessary and sufficient.

We define informativeness in terms of knowledge about the true posterior history. In general, direct and language independent communication strategies are less informative than others. Any element in the relative interior of the set of all language independent communication strategies which induce a particular distribution belongs to the set of least informative communication strategies.

\section{References}

Alonso, R. and Camara, O. (2016): "Bayesian Persuasion with Heterogeneous Priors". In: Journal of Economic Theory 165, pp. 672-706. 
Alonso, R. and Câmara, O. (2016): "Persuading Voters". In: American Economic Review 106(11), pp. 3590-3605.

Arieli, I., Babichenko, Y., Sandomirskiy, F., and Tamuz, O. (2020): "Feasible Joint Posterior Beliefs". arXiv preprint arXiv:2002.11362.

Aumann, R. J. (1976): "Agreeing to Disagree". In: The annals of statistics, pp. 12361239.

Beauchêne, D., Li, J., and Li, M. (2019): "Ambiguous Persuasion". In: Journal of Economic Theory 179, pp. 312-365.

Blackwell, D. (1953): "Equivalent Comparisons of Experiments". In: The annals of mathematical statistics, pp. 265-272.

Ganuza, J.-J. and Penalva, J. S. (2010): "Signal Orderings Based on Dispersion and the Supply of Private Information in Auctions". In: Econometrica 78(3), pp. 1007-1030.

Gentzkow, M. and Kamenica, E. (2016): "Competition in Persuasion". In: The Review of Economic Studies 84(1), pp. 300-322.

Hintikka, J. (1962): Knowledge and Belief: An Introduction to the Logic of the Two Notions. Cornell University Press.

Ichihashi, S. (2019): "Limiting Sender's Information in Bayesian Persuasion". In: Games and Economic Behavior 117, pp. 276-288.

Kamenica, E. and Gentzkow, M. (2011): "Bayesian Persuasion". In: American Economic Review 101, pp. 2590-2615.

Kerman, T., Herings, P., and Karos, D. (2020): "Persuading Strategic Voters". In: Maastricht University, Research Memorandum 20004.

Li, C. (2017): "A Model of Bayesian Persuasion with Transfers". In: Economics Letters 161, pp. 93-95.

Milgrom, P. and Stokey, N. (1982): "Information, Trade and Common Knowledge". In: Journal of Economic Theory 26(1), pp. 17-27.

Osborne, M. J. and Rubinstein, A. (1994): A Course in Game Theory. MIT press.

Rick, A. (2013): "The Benefits of Miscommunication". Working Paper.

Wang, Y. (2013): "Bayesian Persuasion with Multiple Receivers". Working Paper.

Ziegler, G. (2019): Adversarial Bilateral Information Design. Tech. rep. mimeo, Northwestern University. 